\title{
Asymptotically safe SU(5) GUT
}

\author{
M. Fabbrichesi, ${ }^{1}$ C. M. Nieto, ${ }^{2}$ A. Tonero $\odot,{ }^{3}$ and A. Ugolotti ${ }^{4}$ \\ ${ }^{1}$ INFN, Sezione di Trieste, Via Valerio 2, 34127 Trieste, Italy \\ ${ }^{2}$ Universidad Industrial de Santander, Carrera 27 Calle 9, Bucaramanga, Santander, Colombia \\ ${ }^{3}$ Ottawa-Carleton Institute for Physics, Carleton University, \\ 1125 Colonel By Drive, Ottawa, Ontario K1S 5B6, Canada \\ ${ }^{4}$ Theoretisch-Physikalische Institut (TPI), Friedrich-Schiller-Universität, \\ Abbeanum, Fröbelstieg 1, 07743 Jena, Germany
}

(Received 13 January 2021; accepted 1 April 2021; published 24 May 2021)

\begin{abstract}
We minimally extend the Standard Model field content by adding new vectorlike fermions at the TeV scale to allow gauge coupling unification at a realistic scale. We embed the model into an SU(5) grand unified theory that is asymptotically safe and features an interacting fixed point for the gauge coupling. There are no Landau poles of the U(1) gauge and Higgs couplings in this new setting. Gauge, Yukawa, and Higgs couplings are retraced from the fixed point and matched at the grand unification scale to those of the Standard Model rescaled up to the same energy. All couplings, their fixed point values, and critical exponents always remain in the perturbative regime.
\end{abstract}

DOI: 10.1103/PhysRevD.103.095026

\section{INTRODUCTION}

A quantum field theory is asymptotically safe if all its couplings reach a fixed point in the UV limit, as they run along the flow dictated by their renormalization group equations [1,2]. The fixed point can be interacting or free (Gaussian). In the latter case, asymptotic safety (AS) reduces to asymptotic freedom [3,4]. In both cases, we can say that the theory is UV complete because it is well behaved and predictive at all energies.

The Standard Model (SM) is not asymptotically safe because of the uncertain fate of the Higgs boson quartic coupling and the presence of the Landau pole in the $\mathrm{U}(1)_{\mathrm{Y}}$ gauge coupling. In particular, the latter divergence feeds back into the renormalization group (RG) flow of the quartic Higgs self-interaction inducing a Landau pole also in the scalar sector. Furthermore, the Higgs quartic coupling - given the current experimental value of the top mass -becomes negative before the Planck scale, making the electroweak vacuum metastable [5]. Quite in general, for a given cutoff scale and fixed value for the top mass, the Higgs mass has to exceed a lower bound in order to avoid the metastability issue of the scalar potential [6-11]. Although different mechanisms can be devised to solve the problem of the instability of the quartic coupling, the

Published by the American Physical Society under the terms of the Creative Commons Attribution 4.0 International license. Further distribution of this work must maintain attribution to the author(s) and the published article's title, journal citation, and DOI. Funded by SCOAP ${ }^{3}$.
Landau pole of the $\mathrm{U}(1)_{\mathrm{Y}}$ gauge coupling has proved to be a stumbling block.

Should the SM be asymptotically safe? For all practical purposes, the breakdown of the perturbative regime represented by the presence of the Landau pole in the $\mathrm{U}(1)_{\mathrm{Y}}$ gauge coupling can be ignored for it takes place at energies well beyond the Planck scale. Be that as it may, the taming of the $\mathrm{U}(1)_{\mathrm{Y}}$ Landau pole becomes essential if we take the UV behavior as our guidance in searching for a completion of the SM.

A research program based on the safe UV completion of the SM has been actively pursued in recent years thanks to the progress that has been made in gauge theories with a large number of vectorlike fermions (VLF) and gauge bosons - for which it is possible to state rigorous results $[12,13]$ in the Veneziano limit. These findings have encouraged the investigation of the extension with vectorlike fermions of models containing at least some of the features of the SM [14-17] and, more recently, the SM itself $[18,19]$. All the same, as impressive as these results are, it is fair to say that the Landau pole of the $\mathrm{U}(1)_{\mathrm{Y}}$ gauge coupling has proven to be a stumbling block. It appears that all perturbative stable fixed points of the possible extension of the SM with vectorlike fermions only admit a lowenergy matching if the $\mathrm{U}(1)_{\mathrm{Y}}$ gauge coupling vanishes and the theory is trivial in that sector.

This problem requires us to look into viable options that allow us to circumvent the $\mathrm{U}(1)_{\mathrm{Y}}$ triviality problem.

One possible way out has been recently suggested by studying an asymptotically safe version of QED. It has been shown in Ref. [20] that an enlarged theory space-where 
higher-dimensional operators such as a Pauli spin-field coupling are included-opens the possibility for UVcomplete realizations of QED due to the presence of interacting fixed points. Other possible solutions to the triviality problem are obtained by including ad hoc gravitational contributions $[21,22]$ - a procedure with its own conceptual difficulties - or venturing into the nonperturbative regime [19].

In this work, we follow the more conservative choice of looking into a grand unified theory (GUT) extension of the SM [23-27], where the Abelian gauge group is merged into a larger non-Abelian group for which there is no Landau pole to begin with. The possibility of having an asymptotically safe GUT has been discussed in Refs. [28-31]. Other embeddings that solve the $\mathrm{U}(1)_{\mathrm{Y}}$ problem have been proposed in Refs. [32,33].

We study minimal GUT extensions based on the SU(5) gauge group. The specific model we consider below the GUT scale consists in the SM enlarged by the addition of vectorlike fermions - that is, fermions whose right- and left-handed components belong to the same representation of the gauge group and for which a Dirac mass term can be explicitly written. They enter at the scale of $1 \mathrm{TeV}$ and transform under some specific representations of the SM gauge group. We are interested in the extensions of the SM leading to good gauge coupling unification, which can be achieved with both minimal $[34,35]$ or nonminimalVLF multiplicities $[35,36]$.

The role played by the vectorlike fermions is twofold: below the GUT scale, their presence leads to a "good" (that is, around $1 \%$ of relative difference) gauge coupling unification and ensures such unification at a scale which is not constrained by low-energy experiments like proton decay; above the GUT scale, thanks to their new Yukawa interactions, they are crucial for generating nontrivial perturbative UV fixed points for the SU(5) gauge coupling [37].

At this stage, we do not want to classify all the possible models; rather, we intend to show a specific working example as a proof of concept. In particular, we consider one of the possible minimal extensions that were classified in Ref. [34] which presents the same features of a splitsupersymmetry (SUSY) scenario: the LVG model. The corresponding $\mathrm{TeV}$-scale vectorlike fermions are embedded in proper $\mathrm{SU}(5)$ representations at the GUT scale with multiplicities adjusted such that the UV fixed point of the $\mathrm{SU}(5)$ gauge coupling has a numerical value rather close to that of the unification of the three SM gauge couplings at the GUT scale. The gauge couplings run from the electroweak to the GUT scale where they come close to each other and merge into the SU(5) gauge coupling. From the GUT scale on, the unified gauge coupling remains at its fixed point.

The other relevant couplings that are present below the GUT scale, namely, the top-quark Yukawa, the vectorlike fermion Yukawa couplings, and the quartic Higgs coupling, run through the GUT scale where they merge into their SU (5) GUT counterpart and reach their own UV fixed points together with the other couplings of the GUT model (additional vectorlike fermions Yukawa couplings and GUT scalar potential couplings).

All couplings, the fixed point values, and critical exponents always remain in the perturbative regime-a fact that suggests that the fixed point and the renormalization group flow are stable.

\section{TOWARD THE GUT SCALE}

The gauge couplings of the SM run toward each other in a manner that is suggestive of a possible unification. Though they come rather close, they do so at a scale of order $10^{13}-10^{14} \mathrm{GeV}$, that is too low for the GUT theory to be consistent with data on the proton lifetime [38]. On the other hand, it is known that the addition of new charged states can modify the running and move the GUT scale to a higher value. Among the possible models, minimal nonsupersymmetric extensions were discussed in Refs. [34,39-47] and [48-53]. On the other hand, supersymmetric extensions can be found in Refs. [54-59]. Yet, the minimal supersymmetric GUT model with squark masses $m_{\tilde{f}} \lesssim 2 \mathrm{TeV}$ is excluded if one combines the limits on proton decay mediated by the colored Higgs [60] with the constraints obtained by the requirement that the Yukawa couplings do not blow up before Planck scale [61,62].

In this work, we focus on nonsupersymmetric theories and consider one specific minimal extension of the SM that has been classified in Ref. [34], namely, the LVG model, which has the same low-energy field content of the "splitSUSY" scenario, as summarized in Table I. The label " $L$ " stands for vectorlike fermions $\psi_{L}$ that transform under the $(1,2,1 / 2)$ representation of the SM gauge group and have the same quantum numbers of the minimal supersymmetric SM Higgsino. The labels " $V$ " and " $G$ " stand for Majorana fermions $\psi_{V}$ and $\psi_{G}$ that transform, respectively, under the $(1, \mathbf{3}, 0)$ and $(\mathbf{8}, 1,0)$ representations of the SM. The fields $\psi_{V}$ and $\psi_{G}$ are like the wino and gluino fields of the minimal supersymmetric SM. The multiplicities of these beyond the SM representations are all the same and equal to $N_{\mathrm{f}}=1$. These extra matter fields are added at the scale of $1 \mathrm{TeV}$.

TABLE I. Quantum numbers and multiplicities of the vectorlike fermions in the LVG model.

\begin{tabular}{lcccc}
\hline \hline Fields & $\mathrm{SU}(3)_{\mathrm{c}}$ & $\mathrm{SU}(2)_{\mathrm{L}}$ & $\mathrm{U}(1)_{\mathrm{Y}}$ & $N_{\mathrm{f}}$ \\
\hline$\psi_{L}$ & 1 & $\mathbf{2}$ & $1 / 2$ & 1 \\
$\psi_{V}$ & 1 & $\mathbf{3}$ & 0 & 1 \\
$\psi_{G}$ & $\mathbf{8}$ & 1 & 0 & 1 \\
\hline \hline
\end{tabular}


The Lagrangian of the LVG model is given by

$$
\begin{aligned}
\mathcal{L}_{\mathrm{LVG}}= & \mathcal{L}_{\mathrm{SM}}+\bar{\psi}_{L} \mathrm{i} \not \supset \psi_{L}-M_{L} \bar{\psi}_{L} \psi_{L}+\operatorname{Tr} \bar{\psi}_{V} \mathrm{i} \not \supset \psi_{V} \\
& -M_{V} \operatorname{Tr} \bar{\psi}_{V} \psi_{V}+\operatorname{Tr} \bar{\psi}_{G} \mathrm{i} \not \supset \psi_{G}-M_{G} \operatorname{Tr} \bar{\psi}_{G} \psi_{G} \\
& -y_{V} \bar{\psi}_{L} \psi_{V} H+\text { H.c. },
\end{aligned}
$$

where $\psi_{V}=\psi_{V}^{i} T_{i}$, with $T_{i}$ the generators of $\mathrm{SU}(2)_{\mathrm{L}}$ such that $\operatorname{Tr}\left(T_{i} T_{j}\right)=1 / 2 \delta_{i j}$, and $\psi_{G}=\psi_{G}^{a} \lambda_{a}$, with $\lambda_{a}$ the generators of $\mathrm{SU}(3)_{\mathrm{c}}$ normalized again such that $\operatorname{Tr}\left(\lambda_{a} \lambda_{b}\right)=$ $1 / 2 \delta_{a b}$. The indices $(i, j=1,2,3)$ and $(a, b=1,2, \ldots, 8)$ belong to the adjoint representations of $\mathrm{SU}(2)_{\mathrm{L}}$ and $\mathrm{SU}(3)_{\mathrm{c}}$, respectively. Let us notice that the SM Higgs doublet $H$ can couple to the vectorlike fermions via a new Yukawa interaction whose coupling is $y_{V}$.

The SM Lagrangian $\mathcal{L}_{\mathrm{SM}}$ in Eq. (1) is written following the convention used in Ref. [18]; in particular, the Higgs quartic interactions is parametrized as $-\lambda H^{\dagger} H / 2$. We did not include Yukawa interactions that mix SM fermions and vectorlike fermions (this can be achieved by imposing a $\mathbb{Z}_{2} /$ parity-type symmetry, under which SM fermions are even while vectorlike fermions are odd).

\section{A. Renormalization group flow}

In this section, we study the renormalization group flow for the couplings of the LVG model, in what is known as the 211-SCHEME approximation, where the gauge coupling $\beta$ functions are computed at two-loop order while the Yukawa and scalar couplings are computed at one-loop order [63-67]. This scheme is a compromise between the more formally consistent 321-SCHEME-a scheme with $\beta$ functions at the three-loop order in the gauge coupling, two-loop order in the Yukawa couplings, and one-loop order scalar couplings-and the computational manageability of the $\beta$ functions. The simpler and consistent 210SCHEME would not allow us to study the renormalization of the scalar potential. We trust that higher-loop corrections do not significantly change our results since we always work well within the perturbative regime.

We compute the $\beta$ functions in the $\overline{\mathrm{MS}}$ renormalization scheme and consider only the gauge, top-Yukawa, Higgs scalar quartic, and vectorlike fermion Yukawa couplings. In the rest of this paper, we will neglect all other Yukawa couplings in the SM as they are small compared to that of the top quark. Let us define the rescaled couplings $\alpha$,

$\alpha_{i}=\frac{g_{i}^{2}}{(4 \pi)^{2}}, \quad \alpha_{\mathrm{t}}=\frac{y_{\mathrm{t}}^{2}}{(4 \pi)^{2}}, \quad \alpha_{\lambda}=\frac{\lambda}{(4 \pi)^{2}}, \quad \alpha_{V}=\frac{y_{V}^{2}}{(4 \pi)^{2}}$,

where $g_{1}, g_{2}, g_{3}, y_{\mathrm{t}}$, and $\lambda$ are the SM couplings and $y_{V}$ is the vectorlike fermion Yukawa coupling. This definitions are convenient in expressing the $\beta$ functions as polynomials with rational coefficients. Note that the definition of $\alpha_{i}$ for the gauge couplings is different from the usual one by an additional factor of $(4 \pi)$ in the denominator. The $\beta$ functions of the LVG model read

$$
\begin{gathered}
\partial_{t} \alpha_{1}=\beta_{1}^{\mathrm{SM}, \mathrm{NLO}}+\left(\frac{4}{3}+\alpha_{1}+3 \alpha_{2}-6 \alpha_{V}\right) \alpha_{1}^{2}, \\
\partial_{t} \alpha_{2}=\beta_{2}^{\mathrm{SM}, \mathrm{NLO}}+\left(4+\alpha_{1}+59 \alpha_{2}-22 \alpha_{V}\right) \alpha_{2}^{2}, \\
\partial_{t} \alpha_{3}=\beta_{3}^{\mathrm{SM}, \mathrm{NLO}}+\left(4+96 \alpha_{3}\right) \alpha_{3}^{2} \\
\partial_{t} \alpha_{\mathrm{t}}=\beta_{\mathrm{t}}^{\mathrm{SM}, \mathrm{LO}}+12 \alpha_{V} \alpha_{\mathrm{t}} \\
\partial_{t} \alpha_{V}=\left(15 \alpha_{V}+6 \alpha_{\mathrm{t}}-\frac{3}{2} \alpha_{1}-\frac{33}{2} \alpha_{2}\right) \alpha_{V}, \\
\partial_{t} \alpha_{\lambda}=\beta_{\lambda}^{\mathrm{SM}, \mathrm{LO}}+24 \alpha_{V} \alpha_{\lambda}-48 \alpha_{V}^{2}
\end{gathered}
$$

where $\beta_{i}^{\mathrm{SM}, \mathrm{NLO}}, \beta_{\mathrm{t}}^{\mathrm{SM}, \mathrm{LO}}$, and $\beta_{\lambda}^{\mathrm{SM}, \mathrm{LO}}$ are the $\mathrm{SM} \beta$ functions given in the Appendix A. The new terms pertaining to physics beyond the SM are explicitly shown: the vectorlike fermion contributions to the gauge couplings are computed using the formulas in Appendix B. The contributions of the vectorlike fermion Yukawa coupling to the gauge, topYukawa, and Higgs quartic couplings as well as the $\beta$ function of $\alpha_{V}$ itself are computed using the results of Ref. [68]. An explanation of the latter contributions is provided in Appendix B.

In computing the renormalization group flow, we assume that the vectorlike fermions have all the same mass $M_{L}=M_{V}=M_{G}=1 \mathrm{TeV}$. In principle, there are no particular restrictions in choosing, for example, a different mass for each of the three vectorlike fermions. Our particular choice has been made for simplicity. The initial conditions for the SM couplings $\alpha_{i}, \alpha_{\mathrm{t}}$, and $\alpha_{\lambda}$ are given at the $Z$-boson mass $M_{Z}=91.19 \mathrm{GeV}$. These values are shown in Table II and are obtained by using the tree-level relations between the couplings and the SM input experimental values [69]. The renormalization group flow for the couplings of the LVG model, where $\alpha_{V}$ is set to zero, is shown in Fig. 1. The gray vertical line on the left corresponds to the scale at which the vectorlike fermions become dynamical and their presence makes it possible to achieve gauge coupling unification (with a maximum of relative difference of $1.2 \%$ ) at the scale of

TABLE II. Initial conditions at $M_{Z}=91.19 \mathrm{GeV}$ for the SM gauge, top-Yukawa, and Higgs quartic couplings.

\begin{tabular}{lcccc}
\hline \hline $\boldsymbol{\alpha}_{1}$ & $\boldsymbol{\alpha}_{2}$ & $\boldsymbol{\alpha}_{3}$ & $\boldsymbol{\alpha}_{\mathrm{t}}$ & $\boldsymbol{\alpha}_{\lambda}$ \\
\hline 0.0008091 & 0.002689 & 0.009390 & 0.006298 & 0.001634 \\
\hline \hline
\end{tabular}




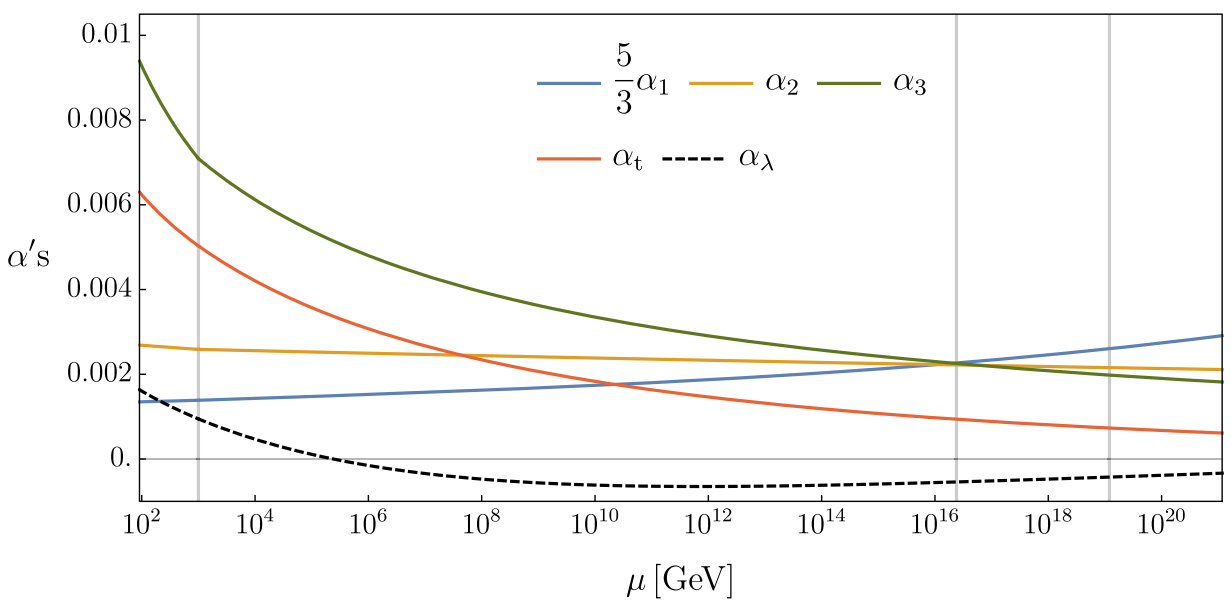

FIG. 1. Renormalization group flow of the SM couplings 5/3 $\alpha_{1}, \alpha_{2}, \alpha_{3}, \alpha_{\mathrm{t}}$, and $\alpha_{\lambda}$ for the LVG model. The initial conditions are given at the scale $M_{Z}$, and the vectorlike fermions are included at the scale of $1 \mathrm{TeV}$ (gray vertical line on the left). The flow is considered up to the Planck scale (gray vertical line on the right). The gray vertical line in the middle corresponds to the GUT scale at which the gauge couplings unify.

$$
M_{\mathrm{GUT}} \simeq 2.399 \times 10^{16} \mathrm{GeV},
$$

which is below the Planck scale, which is highlighted in Fig. 1 by the gray vertical line on the right. The values of the SM couplings at the GUT scale are

$\alpha_{i}\left(M_{\mathrm{GUT}}\right) \simeq 0.002247, \quad \alpha_{\mathrm{t}}\left(M_{\mathrm{GUT}}\right) \simeq 0.0009388$,

$\alpha_{\lambda}\left(M_{\mathrm{GUT}}\right) \simeq-0.0005420$.

These values will represent the IR target which has to be connected with the UV behavior of the model defined at the GUT scale, behavior controlled by the existence of interacting fixed points. The procedure that we use to connect, whenever possible, a UV fixed point with the IR target of Eq. (10) will be exhaustively explained in Sec. V. As already mentioned, at the GUT scale, the LVG model becomes embedded into an $\mathrm{SU}(5)$ gauge theory, such that the SM gauge couplings merge into the SU(5) gauge coupling and the top-Yukawa coupling as well as the Higgs quartic coupling merge into their SU(5) GUT counterparts. The latter couplings have to match the values in Eq. (10) while flowing down from (at least) one fixed point of the SU(5) GUT theory itself.

To understand the sensitivity of the gauge coupling unification on the mass of the vectorlike fermions, we have reported in Table III the variation of the maximum relative difference among the gauge couplings,

$$
\max \left|\Delta_{i j}\right|[\%] \equiv \max \left|\left(\alpha_{i}-\alpha_{j}\right) / \alpha_{i}\right| \cdot 10^{2}
$$

for different values of $M_{L}=M_{V}=M_{G} \equiv M_{\mathrm{LVG}}$ and with the boundary condition $\alpha_{V}=0$. In addition, we have also written in Table III the corresponding values for $\alpha_{\mathrm{GUT}}$ and $M_{\mathrm{GUT}}$. Reducing the mass of the vectorlike fermions allows us to have a better gauge coupling unification at the expense of a higher value of $\alpha_{\mathrm{GUT}}$ and of the unification scale. On the other hand, we fix $M_{\mathrm{LVG}}$ around $1 \mathrm{TeV}$ so as not to worry about current and future exclusion bounds from LHC data.

\section{B. Higgs potential stability}

The problem of the Higgs potential instability is already present in the SM, and we do not provide a solution to this issue in our model with vectorlike fermions. Indeed, the renormalization group flow, as plotted in Fig. 1, shows that the quartic Higgs coupling $\alpha_{\lambda}$ becomes negative at around $10^{5} \mathrm{TeV}$ (in the present 211-SCHEME), signaling a vacuum instability or metastability.

The presence of vectorlike fermions mitigates this problem as they make $\alpha_{\lambda}$ turn negative at higher energies compared to the SM case. This is because $\alpha_{\mathrm{t}}$ runs faster toward zero with respect to the SM case, which is, in turn, due to the fact that $\alpha_{2}$ and $\alpha_{3}$ run slower than in the SM. Yet, considering the field content of our model, we have that the variation of the instability scale with respect to the SM case

TABLE III. List of different values for the mass of the vectorlike fermions $M_{\mathrm{LVG}}$ and corresponding variation of $\alpha_{\mathrm{GUT}}, M_{\mathrm{GUT}}$, and the maximum relative difference between the gauge couplings, i.e., $\max \left|\Delta_{i j}\right|$. The beyond-the-SM Yukawa coupling $\alpha_{V}$ is set to zero.

\begin{tabular}{lccc}
\hline \hline $\boldsymbol{M}_{\mathbf{L V G}}(\mathrm{TeV})$ & $\boldsymbol{\alpha}_{\text {GUT }}$ & $\boldsymbol{m a x}\left|\Delta_{i j}\right|(\%)$ & $\boldsymbol{M}_{\text {GUT }}\left(10^{16} \mathbf{G e V}\right)$ \\
\hline 0.1 & 0.002284 & 0.8460 & 3.804 \\
1 & 0.002247 & 1.188 & 2.399 \\
10 & 0.002211 & 1.554 & 1.553 \\
100 & 0.002178 & 1.928 & 1.023 \\
1000 & 0.002146 & 2.302 & 0.6805 \\
\hline \hline
\end{tabular}


is minimal (about 26\%). In principle, one can think of preventing the quartic Higgs coupling from becoming negative and keeping good gauge coupling unification below the Planck scale, by adding a large number of vectorlike fermions in different SM representations, as shown in Ref. [36]. This choice is, however, not minimal, and its study is beyond the scope of this paper.

In addition, it is also known that the metastability scale in the SM moves to higher values when one considers twoloop and three-loop $\beta$ function for $\alpha_{\lambda}\left(10^{7}\right.$ and $10^{9} \mathrm{GeV}$, respectively), as shown in Refs. [7,8]. In the end, the problem still persists, and one either accepts a metastable potential, as in the SM, or one adds additional fields (for example, vectorlike fermions or also a scalar singlet) so as to prevent the Higgs quartic coupling to become negative before the Planck scale.

The present discussion about the scalar potential stability is largely accepted within the standard lore of perturbation theory, which intimately associates the instability scale to the existence of a lower bound for the Higgs mass. However, the presence of such an instability scale becomes questionable as soon as one adopts different approaches. For example, lattice simulations have shown that the lower bound for the Higgs mass merely arises from consistency conditions imposed on the bare action and no reference to a low-energy stability issue has to be made [70-77]. The same point of view got also substantiated by functional methods, showing that the conventional lower Higgs-mass bound can even be relaxed given an appropriate consistent definition of the bare action [21,78-85].

\section{ABOVE THE GUT SCALE}

Aiming at an asymptotically safe scenario, we now focus our analysis beyond the GUT scale, where we assume that an underlying SU(5) symmetry is restored. Consequently, the vectorlike fermions of the LVG model discussed in the previous section are embedded into proper multiples of $\mathrm{SU}(5)$, just like is the case for the SM fields. This unification group choice seems to be the most natural since SU(5) not only can play the role of a self-contained unified gauge symmetry [23] but also show up in breaking chains of larger GUT groups. The Lagrangian of the SU(5) SM GUT theory is given by [86]

$$
\begin{aligned}
\mathcal{L}_{\mathrm{SM}}^{\mathrm{GUT}}= & -\frac{1}{4} F_{\mu \nu}^{a} F_{a}^{\mu \nu}+\bar{\chi}_{\overline{5}} \mathrm{i} \not \not \chi_{\overline{5}}+\frac{1}{2} \operatorname{Tr}\left(\bar{\chi}_{10} \mathrm{i} \not D \chi_{10}\right) \\
& +\operatorname{Tr}\left[\left(D_{\mu} \Sigma\right)^{\dagger}\left(D^{\mu} \Sigma\right)\right]+\left(D_{\mu} \Phi\right)^{\dagger}\left(D^{\mu} \Phi\right)-V(\Phi, \Sigma) \\
& -y_{\mathrm{b}} \bar{\chi}_{\overline{5}}^{c} \chi_{10} \Phi^{*}+\frac{y_{\mathrm{t} 10}}{8} \varepsilon_{5} \bar{\chi}_{10}^{c} \chi_{10} \Phi+\text { H.c. },
\end{aligned}
$$

where $F_{\mu \nu}^{a}$ is the field strength of the $\mathrm{SU}(5)$ gauge fields $A_{\mu}^{a}(a=1, \ldots, 24)$, which include the SM gluons, the electroweak gauge bosons, and the heavy GUT gauge bosons.
The right-handed down quarks and the left-handed lepton doublets are embedded into the left-handed field $\chi_{\overline{5}}$ transforming as an antifundamental $\overline{\mathbf{5}}$ representation of $\mathrm{SU}(5)$, while the left-handed quark doublets, right-handed up quarks, and leptons are embedded into the left-handed field $\chi_{10}$ transforming as an antisymmetric 10 representation of SU(5). The charge conjugation of a fermionic field is expressed by a superscript $c$, for example, $\chi_{\overline{5}}^{c}=\mathcal{C}\left(\bar{\chi}_{\overline{5}}\right)^{\mathrm{T}}$, where $\mathcal{C}$ is the charge conjugation operator.

The Higgs field is embedded into $\Phi$, transforming as a fundamental 5 representation of SU(5). Even though the fermionic matter content of the SM can be fitted entirely into the $\overline{\mathbf{5}}$ and $\mathbf{1 0}$ representations of SU(5), the scalar sector is extended by an adjoint scalar field $\Sigma$, in the $\mathbf{2 4}$ representation, which is needed in order to break the SU(5) into the SM gauge group.

The Lagrangian in Eq. (12) includes only the top- and bottom-Yukawa couplings, which are the most relevant ones. We set to zero the Yukawa couplings of the first and second generations. The fermionic field $\chi_{10}$ is a $5 \times 5$ antisymmetric matrix such that the corresponding Yukawa interaction is constructed by mean of the Levi-Civita tensor in five dimensions, i.e., $\varepsilon_{5}$. In terms of components, the Yukawa interaction term is $\varepsilon_{5} \bar{\chi}_{10}^{c} \chi_{10} \Phi=\varepsilon_{i j k l m} \bar{\chi}_{10}^{c i j} \chi_{10}^{k l} \Phi^{m}$.

The quartic terms of the scalar potential $V(\Phi, \Sigma)$ in Eq. (12) read [86]

$$
\begin{aligned}
V^{\text {quartic }}(\Phi, \Sigma)= & \frac{\lambda_{\Sigma}}{2} \operatorname{Tr}\left(\Sigma^{4}\right)+\frac{\lambda_{\Sigma}^{\prime}}{2}\left[\operatorname{Tr}\left(\Sigma^{2}\right)\right]^{2}+\frac{\lambda_{H}}{2}\left(\Phi^{\dagger} \Phi\right)^{2} \\
& +2 \lambda_{H \Sigma} \Phi^{\dagger} \Phi \operatorname{Tr}\left(\Sigma^{2}\right)+2 \lambda_{H \Sigma}^{\prime} \Phi^{\dagger} \Sigma^{2} \Phi,
\end{aligned}
$$

where $\lambda_{X}=\left(\lambda_{\Sigma}, \lambda_{\Sigma}^{\prime}, \lambda_{H}, \lambda_{H \Sigma}\right)$ are the quartic scalar couplings of the GUT model.

At the unification scale, the vectorlike fermions of the LVG model in Table I are embedded into proper multiples of SU(5), see Table IV. We assume that the field $\psi_{L}$ gets embedded into vectorlike fermions $\Psi_{5}$, with multiplicity $N_{5} \geq 1$, transforming under the fundamental representation of SU(5), while $\psi_{V}$ and $\psi_{G}$ get embedded into Majorana fermions $\Psi_{24}$, with multiplicity $N_{24} \geq 1$, transforming under the adjoint representation of SU(5). ${ }^{1}$

Therefore, the Lagrangian of the LVG GUT model is

$$
\begin{aligned}
\mathcal{L}_{\mathrm{LVG}}^{\mathrm{GUT}}= & \mathcal{L}_{\mathrm{SM}}^{\mathrm{GUT}}+\bar{\Psi}_{5}^{I} \mathrm{i} \not \not \Psi_{5}^{I}-M_{5} \bar{\Psi}_{5}^{I} \Psi_{5}^{I}+\operatorname{Tr}\left(\bar{\Psi}_{24}^{J} \mathrm{i} \not \not \Psi_{24}^{J}\right) \\
& -M_{24} \operatorname{Tr}\left(\bar{\Psi}_{24}^{J} \Psi_{24}^{J}\right)-y_{5 \Sigma} \bar{\Psi}_{5}^{I} \Sigma \Psi_{5}^{I} \\
& -y_{24 \Sigma} \operatorname{Tr}\left(\Sigma \bar{\Psi}_{24}^{J} \Psi_{24}^{J}\right)-y_{\nu} \sum_{I, J}\left(\bar{\Psi}_{5}^{I} \Psi_{24}^{J} \Phi+\text { H.c. }\right)
\end{aligned}
$$

\footnotetext{
${ }^{1}$ The fundamental and adjoint representations of SU(5) can be decomposed under the SM gauge group as follows (see, for example, Ref. [87]): $\mathbf{5}=(1, \mathbf{2}, 1 / 2) \oplus(\mathbf{3}, 1,-1 / 3)$ and $\mathbf{2 4}=$ $(1,1,0) \oplus(1, \mathbf{3}, 0) \oplus(\mathbf{8}, 1,0) \oplus(\mathbf{3}, \mathbf{2},-5 / 6) \oplus(\overline{\mathbf{3}}, \mathbf{2}, 5 / 6)$.
} 
TABLE IV. Quantum numbers and multiplicities of the vectorlike fermions in the LVG GUT model.

\begin{tabular}{lcc}
\hline \hline Fields & $\mathrm{SU}(5)$ & $N_{\mathrm{f}}$ \\
\hline$\Psi_{5}$ & $\mathbf{5}$ & $N_{5}$ \\
$\Psi_{24}$ & $\mathbf{2 4}$ & $N_{24}$ \\
\hline \hline
\end{tabular}

where $I$ and $J$ run over the flavor numbers, i.e., $I=$ $1, \ldots, N_{5}$ and $J=1, \ldots, N_{24}$. In the LVG GUT Lagrangian of the latter equation, we have introduced three Yukawa couplings involving the vectorlike fermions: $y_{5 \Sigma}, y_{24 \Sigma}$, and $y_{\nu}$. The first two terms in the second line respect an $\mathrm{U}\left(N_{5}\right) \times \mathrm{O}\left(N_{24}\right)$ flavor symmetry which is explicitly broken by $y_{\nu}$ interactions. In principle, additional gaugeinvariant Yukawa terms are possible; however, we restrict the number of such interactions to just 3 by imposing a $\mathbb{Z}_{2} /$ parity-type symmetry, under which the SM fermions are even while the vectorlike fermions are odd.

\section{A. $\beta$ functions}

In this section, we compute the $\beta$ functions of the LVG GUT model given in Eq. (14). We adopt again the $\overline{\mathrm{MS}}$ renormalization scheme and the 211-SCHEME regarding the loop orders. Let us first define the rescaled couplings $\alpha$ 's,

$$
\alpha_{5}=\frac{g_{5}}{4 \pi}, \quad \alpha_{X}=\frac{\lambda_{X}}{(4 \pi)^{2}}, \quad \alpha_{z}=\frac{y_{z}}{4 \pi},
$$

where $g_{5}$ is the $\mathrm{SU}(5)$ gauge coupling, $\lambda_{X}$ denotes the couplings in the scalar potential $V^{\text {quartic }}(\Phi, \Sigma)$, and $y_{z}=$ $\left(y_{\mathrm{t} 10}, y_{5 \Sigma}, y_{24 \Sigma}, y_{\nu}\right)$ represents all Yukawa couplings. Note that, differently from the previous Sec. II A, here we define all couplings $\alpha$ 's as linear with respect to the couplings in Eqs. (12)-(14). These definitions allow us to write the $\beta$ functions as polynomials in all the $\alpha$ 's, as is made clear by the equations below. In the 211-SCHEME, the gauge-Yukawa subsystem is closed, and its $\beta$ functions are

$$
\begin{gathered}
\partial_{t} \alpha_{5}=-\frac{40-2 N_{5}-10 N_{24}}{3} \alpha_{5}^{3}-\frac{1184-322 N_{5}-2000 N_{24}}{15} \alpha_{5}^{5} \\
-\left(\frac{9}{2} \alpha_{\mathrm{t} 10}^{2}+\frac{12}{5} N_{5} \alpha_{5 \Sigma}^{2}+\frac{21}{4} N_{24} \alpha_{24 \Sigma}^{2}+\frac{37}{5} N_{5} N_{24} \alpha_{\nu}^{2}\right) \alpha_{5}^{3} \\
\partial_{t} \alpha_{\mathrm{t} 10}=\left(6 \alpha_{\mathrm{t} 10}^{2}+\frac{24}{5} N_{5} N_{24} \alpha_{\nu}^{2}-\frac{108}{5} \alpha_{5}^{2}\right) \alpha_{\mathrm{t} 10} \\
\partial_{t} \alpha_{5 \Sigma}=\left(\frac{11+5 N_{5}}{5} \alpha_{5 \Sigma}^{2}+\frac{21}{20} N_{24} \alpha_{24 \Sigma}^{2}+\frac{12}{5} N_{24} \alpha_{\nu}^{2}-\frac{72}{5} \alpha_{5}^{2}\right) \alpha_{5 \Sigma}+\frac{21}{10} N_{24} \alpha_{\nu}^{2} \alpha_{24 \Sigma}, \\
\partial_{t} \alpha_{24 \Sigma}=\left(\frac{34+21 N_{24}}{20} \alpha_{24 \Sigma}^{2}+N_{5} \alpha_{5 \Sigma}^{2}+N_{5} \alpha_{\nu}^{2}-30 \alpha_{5}^{2}\right) \alpha_{24 \Sigma}+2 N_{5} \alpha_{\nu}^{2} \alpha_{5 \Sigma} \\
\partial_{t} \alpha_{\nu}=\left(\frac{63}{10} N_{5} N_{24} \alpha_{\nu}^{2}+3 \alpha_{\mathrm{t} 10}^{2}+\frac{21}{40} \alpha_{24 \Sigma}^{2}+\frac{6}{5} \alpha_{5 \Sigma}^{2}+\frac{21}{10} \alpha_{24 \Sigma} \alpha_{5 \Sigma}-\frac{111}{5} \alpha_{5}^{2}\right) \alpha_{\nu} .
\end{gathered}
$$

The choice of defining all the $\alpha$ 's as linear with respect to the original couplings becomes clear from the last term in Eq. (18) and Eq. (19). These two contributions arise from the mixed Yukawa interaction among $\Psi_{5}$ and $\Psi_{24}$ [last term in Eq. (14)]. In case we were defining $\alpha_{5 \Sigma}$ and $\alpha_{24 \Sigma}$ as quadratic in $y_{5 \Sigma}$ and $y_{24 \Sigma}$, the last term in Eq. (18) and Eq. (19) would have involved the square root of $\alpha_{5 \Sigma}$ and $\alpha_{24 \Sigma}$, rendering some of the fixed points in Table V inaccessible. For homogeneity, we kept linear also the other Yukawa couplings as well as the gauge coupling. The $\beta$ functions of the scalar couplings are

$$
\begin{gathered}
\partial_{t} \alpha_{\Sigma}=\bar{\beta}_{\Sigma}+\left(4 N_{5} \alpha_{5 \Sigma}^{2}+\frac{21}{5} N_{24} \alpha_{24 \Sigma}^{2}\right) \alpha_{\Sigma}-4 N_{5} \alpha_{5 \Sigma}^{4}+\frac{7}{20} N_{24} \alpha_{24 \Sigma}^{4} \\
\partial_{t} \alpha_{\Sigma}^{\prime}=\bar{\beta}_{\Sigma}^{\prime}+\left(4 N_{5} \alpha_{5 \Sigma}^{2}+\frac{21}{5} N_{24} \alpha_{24 \Sigma}^{2}\right) \alpha_{\Sigma}^{\prime}-\frac{91}{100} N_{24} \alpha_{24 \Sigma}^{4} \\
\partial_{t} \alpha_{H}=\bar{\beta}_{H}+\left(12 \alpha_{\mathrm{t} 10}^{2}+\frac{96}{5} N_{5} N_{24} \alpha_{\nu}^{2}\right) \alpha_{H}-12 \alpha_{\mathrm{t} 10}^{4}-\frac{264}{25} N_{5}^{2} N_{24}^{2} \alpha_{\nu}^{4},
\end{gathered}
$$


TABLE V. List of all fixed points for the LVG GUT model which satisfy the conditions in Eq. (35), for various multiplicities of the vectorlike fermions $\Psi_{24}$ and $\Psi_{5}$. Those fixed points which possess physical trajectories that can be matched with the LVG model at the GUT scale are in bold. These fixed points provide therefore an asymptotically safe SU(5) GUT completion of the SM. Each fixed point $\mathrm{FP}_{n}$ has a certain degeneracy (in the sense that physical properties such as the number of relevant/irrelevant directions as well as the critical exponents are identical) due to the fact that the couplings $\alpha_{\mathrm{t} 10}, \alpha_{24 \Sigma}, \alpha_{5 \Sigma}$, and $\alpha_{\nu}$ can have both positive or negative sign. While $\alpha_{\mathrm{t} 10}$ and $\alpha_{\nu}$ can each be positive or negative independently on the sign of the other coupling, $\alpha_{24 \Sigma}$ and $\alpha_{5 \Sigma}$ have always opposite signs. As an example, $\mathrm{FP}_{1}$ incorporates four degenerate fixed points, while $\mathrm{FP}_{3}$ encodes eight degenerate fixed points.

\begin{tabular}{|c|c|c|c|c|c|c|c|c|c|c|c|}
\hline & $\left(N_{24}, N_{5}\right)$ & $\alpha_{5}$ & $\left|\alpha_{\mathrm{t} 10}\right|$ & $\alpha_{H}$ & $\alpha_{\Sigma}$ & $\alpha_{\Sigma}^{\prime}$ & $\alpha_{H \Sigma}$ & $\alpha_{H \Sigma}^{\prime}$ & $\alpha_{24 \Sigma}$ & $\alpha_{5 \Sigma}$ & $\left|\alpha_{\nu}\right|$ \\
\hline $\mathrm{FP}_{1}$ & $(3,4)$ & 0.04818 & $\mathbf{0}$ & 0.003807 & -0.01001 & 0.004971 & $-4.727 \times 10^{-5}$ & 0.001967 & $\mp 0.1161$ & \pm 0.02663 & 0.02574 \\
\hline $\mathrm{FP}_{2}$ & $(3,4)$ & 0.04818 & 0 & 0.003830 & -0.007946 & 0.004625 & $-1.903 \times 10^{-5}$ & 0.001783 & $\mp 0.1161$ & \pm 0.02663 & 0.02574 \\
\hline $\mathrm{FP}_{3}$ & $(3,4)$ & 0.04831 & 0.05823 & 0.003209 & -0.01088 & 0.005211 & $-1.340 \times 10^{-5}$ & 0.001499 & $\mp 0.1172$ & \pm 0.02306 & 0.02285 \\
\hline $\mathrm{FP}_{4}$ & $(3,4)$ & 0.04831 & 0.05823 & 0.003234 & -0.007527 & 0.004646 & $1.596 \times 10^{-5}$ & 0.001267 & $\mp 0.1172$ & \pm 0.02306 & 0.02285 \\
\hline $\mathrm{FP}_{5}$ & $(3,3)$ & 0.07092 & $\mathbf{0}$ & 0.008350 & -0.02300 & 0.01093 & $-1.194 \times 10^{-4}$ & 0.004450 & 干 0.1709 & \pm 0.04667 & 0.04414 \\
\hline $\mathrm{FP}_{6}$ & $(3,3)$ & 0.07092 & 0 & 0.008428 & -0.01600 & 0.009764 & $-2.651 \times 10^{-5}$ & 0.003832 & $\mp 0.1709$ & \pm 0.04667 & 0.04414 \\
\hline $\mathrm{FP}_{7}$ & $(3,3)$ & 0.07111 & 0.08320 & 0.007029 & -0.02420 & 0.01131 & $-4.575 \times 10^{-5}$ & 0.003450 & $\mp 0.1724$ & \pm 0.04149 & 0.03959 \\
\hline $\mathrm{FP}_{8}$ & $(3,3)$ & 0.07111 & 0.08320 & 0.007096 & -0.01565 & 0.009888 & $3.024 \times 10^{-5}$ & 0.002849 & $\mp 0.1724$ & \pm 0.04149 & 0.03959 \\
\hline $\mathrm{FP}_{9}$ & $(3,3)$ & 0.06178 & 0.1172 & -0.01243 & -0.009077 & 0.005366 & $9.564 \times 10^{-5}$ & 0.002040 & 0 & \pm 0.1028 & 0 \\
\hline $\mathrm{FP}_{10}$ & $(3,3)$ & 0.06178 & 0.1172 & -0.01247 & -0.007685 & 0.003151 & $-4.045 \times 10^{-5}$ & 0.001966 & 0 & \pm 0.1028 & 0 \\
\hline $\mathrm{FP}_{11}$ & $(3,2)$ & 0.09081 & $\mathbf{0}$ & 0.01391 & -0.04006 & 0.01820 & $-1.843 \times 10^{-4}$ & 0.007670 & $\mp 0.2193$ & $\pm \mathbf{0 . 0 7 4 2 9}$ & 0.07003 \\
\hline $\mathrm{FP}_{12}$ & $(3,2)$ & 0.09081 & 0 & 0.01409 & -0.02420 & 0.01561 & $1.125 \times 10^{-5}$ & 0.006293 & $\mp 0.2193$ & \pm 0.07429 & 0.07003 \\
\hline $\mathrm{FP}_{13}$ & $(3,2)$ & 0.09103 & 0.1018 & 0.01173 & -0.04121 & 0.01864 & $-8.246 \times 10^{-5}$ & 0.006132 & $\mp 0.2207$ & \pm 0.06811 & 0.06368 \\
\hline $\mathrm{FP}_{14}$ & $(3,2)$ & 0.09103 & 0.1018 & 0.01187 & -0.02418 & 0.01585 & $6.656 \times 10^{-5}$ & 0.004912 & $\mp 0.2207$ & \pm 0.06811 & 0.06368 \\
\hline $\mathrm{FP}_{15}$ & $(3,2)$ & 0.07586 & $\mathbf{0}$ & 0.005074 & -0.01019 & 0.003843 & $7.419 \times 10^{-4}$ & 0.001431 & $\mathbf{0}$ & \pm 0.1405 & $\mathbf{0}$ \\
\hline $\mathrm{FP}_{16}$ & $(3,2)$ & 0.07586 & $\mathbf{0}$ & 0.003008 & -0.01014 & 0.003722 & $5.230 \times 10^{-4}$ & 0.001366 & $\mathbf{0}$ & $\pm \mathbf{0}$ & $\mathbf{0}$ \\
\hline $\mathrm{FP}_{17}$ & $(3,2)$ & 0.07769 & 0.1474 & 0.01375 & -0.01073 & 0.008801 & -0.003407 & 0.008809 & 0 & \pm 0.1439 & 0 \\
\hline $\mathrm{FP}_{18}$ & $(3,2)$ & 0.07769 & 0.1474 & -0.01965 & -0.01399 & 0.01032 & $3.656 \times 10^{-4}$ & 0.002812 & 0 & \pm 0.1439 & 0 \\
\hline $\mathrm{FP}_{19}$ & $(3,2)$ & 0.07769 & 0.1474 & -0.01976 & -0.01043 & 0.003726 & $-6.145 \times 10^{-5}$ & $2.582 \times 10^{-5}$ & 0 & \pm 0.1439 & 0 \\
\hline $\mathrm{FP}_{20}$ & $(3,2)$ & 0.07769 & 0.1474 & 0.01439 & -0.01016 & 0.004212 & -0.002552 & 0.004854 & 0 & \pm 0.1439 & 0 \\
\hline $\mathrm{FP}_{21}$ & $(3,2)$ & 0.07769 & 0.1474 & -0.01805 & -0.007693 & 0.004046 & $1.309 \times 10^{-5}$ & 0.01010 & 0 & \pm 0.1439 & 0 \\
\hline $\mathrm{FP}_{22}$ & $(3,2)$ & 0.07769 & 0.1474 & -0.01812 & -0.01007 & 0.007458 & $6.199 \times 10^{-4}$ & 0.008703 & 0 & \pm 0.1439 & 0 \\
\hline $\mathrm{FP}_{23}$ & $(3,1)$ & 0.08966 & $\mathbf{0}$ & 0.008210 & -0.009327 & 0.003901 & $8.997 \times 10^{-4}$ & 0.001821 & $\mathbf{0}$ & $\pm \mathbf{0 . 1 9 0 2}$ & $\mathbf{0}$ \\
\hline $\mathrm{FP}_{24}$ & $(3,1)$ & 0.08966 & $\mathbf{0}$ & 0.003731 & -0.009304 & 0.003791 & $5.763 \times 10^{-4}$ & 0.001724 & $\mathbf{0}$ & $\pm \mathbf{0 . 1 9 0 2}$ & $\mathbf{0}$ \\
\hline $\mathrm{FP}_{25}$ & $(3,1)$ & 0.09193 & 0.1744 & 0.02049 & -0.01231 & 0.01458 & -0.002568 & 0.007556 & 0 & \pm 0.1950 & 0 \\
\hline $\mathrm{FP}_{26}$ & $(3,1)$ & 0.09193 & 0.1744 & -0.02423 & -0.005183 & 0.005420 & $5.019 \times 10^{-4}$ & 0.01569 & 0 & \pm 0.1950 & 0 \\
\hline $\mathrm{FP}_{27}$ & $(3,1)$ & 0.09193 & 0.1744 & -0.02771 & -0.009640 & 0.003870 & $-4.388 \times 10^{-5}$ & 0.003042 & 0 & \pm 0.1950 & 0 \\
\hline $\mathrm{FP}_{28}$ & $(3,1)$ & 0.09193 & 0.1744 & 0.02038 & -0.009538 & 0.004422 & -0.003083 & 0.004755 & 0 & \pm 0.1950 & 0 \\
\hline $\mathrm{FP}_{29}$ & $(3,1)$ & 0.09193 & 0.1744 & -0.02436 & -0.007439 & 0.009273 & 0.001347 & 0.01358 & 0 & \pm 0.1950 & 0 \\
\hline $\mathrm{FP}_{30}$ & $(3,1)$ & 0.09193 & 0.1744 & -0.02748 & -0.01349 & 0.0149 & $7.647 \times 10^{-4}$ & $3.547 \times 10^{-3}$ & 0 & \pm 0.1950 & 0 \\
\hline $\mathrm{FP}_{31}$ & $(3,1)$ & 0.1105 & 0.1143 & 0.01787 & -0.06448 & 0.02797 & $4.877 \times 10^{-5}$ & 0.01015 & $\mp 0.2691$ & \pm 0.1161 & 0.1135 \\
\hline $\mathrm{FP}_{32}$ & $(3,1)$ & 0.1105 & 0.1143 & 0.01818 & -0.03263 & 0.02284 & $2.830 \times 10^{-4}$ & 0.007828 & $\mp 0.2691$ & \pm 0.1161 & 0.1135 \\
\hline $\mathrm{FP}_{33}$ & $(3,1)$ & 0.1103 & $\mathbf{0}$ & 0.02095 & -0.06365 & 0.02758 & $-2.092 \times 10^{-5}$ & 0.01210 & $\mp 0.2682$ & $\pm \mathbf{0 . 1 2 2 3}$ & 0.1224 \\
\hline $\mathrm{FP}_{34}$ & $(3,1)$ & 0.1103 & 0 & 0.02133 & -0.03237 & 0.02257 & $2.833 \times 10^{-4}$ & 0.009462 & $\mp 0.2682$ & \pm 0.1223 & 0.1224 \\
\hline $\mathrm{FP}_{35}$ & $\left(N_{24}, N_{5}\right)$ & 0 & 0 & 0 & 0 & 0 & 0 & 0 & 0 & 0 & 0 \\
\hline
\end{tabular}

$$
\begin{aligned}
\partial_{t} \alpha_{H \Sigma}= & \bar{\beta}_{H \Sigma}+\left(6 \alpha_{\mathrm{t} 10}^{2}+2 N_{5} \alpha_{5 \Sigma}^{2}+\frac{21}{10} N_{24} \alpha_{24 \Sigma}^{2}+\frac{48}{5} N_{5} N_{24} \alpha_{\nu}^{2}\right) \alpha_{H \Sigma} \\
& -N_{5} N_{24}\left(2 \alpha_{5 \Sigma}^{2}+\frac{29}{50} \alpha_{24 \Sigma}^{2}+\alpha_{5 \Sigma} \alpha_{24 \Sigma}\right) \alpha_{\nu}^{2}, \\
\partial_{t} \alpha_{H \Sigma}^{\prime}= & \bar{\beta}_{H \Sigma}^{\prime}+\left(6 \alpha_{\mathrm{t} 10}^{2}+2 N_{5} \alpha_{5 \Sigma}^{2}+\frac{21}{10} N_{24} \alpha_{24 \Sigma}^{2}+\frac{48}{5} N_{5} N_{24} \alpha_{\nu}^{2}\right) \alpha_{H \Sigma}^{\prime} \\
& -N_{5} N_{24}\left(-\frac{2}{5} \alpha_{5 \Sigma}^{2}+\frac{13}{10} \alpha_{24 \Sigma}^{2}-\frac{4}{5} \alpha_{5 \Sigma} \alpha_{24 \Sigma}\right) \alpha_{\nu}^{2},
\end{aligned}
$$


where the pure gauge e scalar contributions $\bar{\beta}_{\Sigma}, \bar{\beta}_{\Sigma}^{\prime}, \bar{\beta}_{H}$, $\bar{\beta}_{H \Sigma}$, and $\bar{\beta}_{H \Sigma}^{\prime}$ are given in Appendix C. In particular, in Appendix $\mathrm{C} 1$, we also give a thorough explanation on how we have derived the extra contributions to $\partial_{t} \alpha_{X}$ arising from the presence of the vectorlike fermion Yukawa couplings. In Appendix C 2, we also give a derivation of the $\beta$ functions for the gauge-Yukawa subsystem.

\section{B. Multiplet mass splitting}

At the GUT scale, the vectorlike fermion fields $\psi_{L}, \psi_{V}$, and $\psi_{G}$ are embedded into $\Psi_{5}$ and $\Psi_{24}$ multiplets, as described in the previous section. These SU(5) multiplets contain extra fields, and therefore one has to devise a mechanism that, after SU(5) breaking, keeps these extra components at the GUT scale and allows the $\psi_{L}, \psi_{V}$, and $\psi_{G}$ fields to acquire a mass of order $1 \mathrm{TeV}$. A viable mechanism to achieve this result consists in fine tuning the mass of one particular copy of $\Psi_{5}$, for instance, the one with $I=\omega$. Moreover, the Lagrangian in Eq. (14) should be slightly modified by breaking the $\mathrm{U}\left(N_{5}\right)$ symmetry of the mass term and replacing it with a more general term such as $M_{5}{ }^{I} \bar{\Psi}_{5}{ }^{I} \Psi_{5}{ }^{I}$. Since each of the $\Psi_{5}$ is a direct sum of an $\mathrm{SU}(2)$ doublet and an SU(3) triplet, we can think of the $\psi_{L}$ field as if it were embedded into the $\omega$ copy, namely, $\Psi_{5}^{\omega}=\psi_{L} \oplus \psi_{3}^{\omega}$, where $\psi_{3}^{\omega}$ is the triplet partner. After SU(5) breaking due to a nonzero vacuum expectation value for the adjoint scalar field, i.e., $\langle\Sigma\rangle=f \cdot \operatorname{diag}(2,2,2,-3,-3)$, the contributions to the mass term for the $\omega$ field are

$$
-\left(M_{5}^{\omega}+2 f y_{5 \Sigma}\right) \bar{\psi}_{\mathbf{3}}^{\omega} \psi_{\mathbf{3}}^{\omega}-\left(M_{5}^{\omega}-3 f y_{5 \Sigma}\right) \bar{\psi}_{L} \psi_{L} .
$$

Therefore, the doublet-triplet splitting can be achieved by assuming a cancellation between the two terms contributing to the mass of $\psi_{L}$ with a fine-tuning of one part in $10^{13}$. This fine-tuning procedure is not imposed on the other $N_{5}-1$ copies as they should stay at the GUT scale instead.

A similar mechanism has to be realized also in the case of the $\psi_{V}$ and $\psi_{G}$ embedding into $\Psi_{24}$. Furthermore, the case of the $\psi_{L}$ embedding is analogous to the doublettriplet splitting of the Higgs multiplet in minimal SU(5) GUT models. The simplest solution to the Higgs doublettriplet splitting problem is similar to the one in Eq. (27) and requires a $10^{-14}$ tuning, but it is also possible to devise alternative mechanisms that alleviate this tuning, like the sliding singlet mechanism [88-91].

\section{FIXED POINTS}

Consider a theory characterized by a set of dimensionless couplings $\alpha_{\mathrm{i}}$. The renormalization group flow is completely determined by their $\beta$ functions

$$
\beta_{\mathrm{i}}\left(\alpha_{\mathrm{j}}\right) \equiv \frac{\partial \alpha_{\mathrm{i}}}{\partial t},
$$

where $t=\log \left(\mu / \mu_{0}\right)$ is the logarithm of the sliding scale of the quantum theory. A fixed point of the theory $\alpha_{\mathrm{j}}^{*}$ is defined by the vanishing of the $\beta$ functions of all couplings

$$
\beta_{\mathrm{i}}\left(\alpha_{\mathrm{j}}^{*}\right)=0 .
$$

When the couplings $\alpha_{\mathrm{j}}$ assume the values $\alpha_{\mathrm{j}}^{*}$, the renormalization of the quantum theory stops. In general, a given fixed point can be reached either in the UV or in the IR limit, depending on the direction of the approaching trajectory. Notice that, in the common lore, the distinction between UV and IR fixed points is only meaningful when there is a single coupling in the theory. In the case of more couplings, this distinction becomes unambiguous only if, given two fixed points, it exists an RG trajectory connecting the two of them.

The $\beta$ function of a single coupling is independent of the gauge choice in dimensional regularization. It is regularization scheme independent up to next-to-leading order (NLO). If there are several couplings running together, their $\beta$ functions depend on the scheme already at the NLO [92]. There is therefore a degree of ambiguity in the position of the fixed points because their position could be moved by changing the scheme. We assume that these changes are small if the fixed point is found within the perturbative regime. One should, however, bear in mind this problem of scheme dependence in all the discussions to follow.

Once we have a candidate fixed point, we can study the flow in its immediate neighborhood. We move away from the fixed point and study what happens when we shift the couplings by a small amount $\delta \alpha_{\mathrm{i}} \equiv \alpha_{\mathrm{i}}-\alpha_{\mathrm{i}}^{*}$. To this end, we linearize the $\beta$ functions in the vicinity of the fixed point as

$$
\frac{\partial}{\partial t} \delta \alpha_{\mathrm{i}}=M_{\mathrm{ij}} \delta \alpha_{\mathrm{j}}
$$

and ignore $\mathcal{O}\left(\delta \alpha^{2}\right)$ terms. The quantity

$$
\left.M_{\mathrm{ij}} \equiv \frac{\partial \beta_{\mathrm{i}}}{\partial \alpha_{\mathrm{j}}}\right|_{\alpha_{\mathrm{i}}^{*}}
$$

is referred to as the stability matrix. Next, we can diagonalize the linear system by means of a similarity transformation

$$
\left(S^{-1}\right)_{\mathrm{ij}} M_{\mathrm{j} 1} S_{\ln }=\delta_{\mathrm{in}} \theta_{\mathrm{n}},
$$

where the eigenvalues $\theta_{\mathrm{n}}$ are also known as critical exponents (see the equation below). Defining $z_{\mathrm{i}}=$ $\left(S^{-1}\right)_{\mathrm{ij}} \delta \alpha_{\mathrm{j}}$, we have that the $\beta$ functions and their solutions can be written in the following simplified form:

$$
\frac{\partial z_{\mathrm{i}}}{\partial t}=\theta_{\mathrm{i}} z_{\mathrm{i}} \Rightarrow z_{\mathrm{i}}(t)=c_{\mathrm{i}} \mathrm{e}^{\theta_{\mathrm{i}} t}=c_{\mathrm{i}}\left(\frac{\mu}{\mu_{0}}\right)^{\theta_{\mathrm{i}}} .
$$


From the expression of $z_{\mathrm{i}}$ as functions of $\mu$, we see that there are different situations depending on the sign of $\theta_{\mathrm{i}}$ :

(i) For $\theta_{\mathrm{i}}>0$, as we increase $\mu$ we move away from the fixed point and $z_{\mathrm{i}}$ increases without control; the direction $z_{\mathrm{i}}$ is said to be irrelevant.

(ii) If $\theta_{\mathrm{i}}<0$, as we increase $\mu$, we approach the fixed point; the direction $z_{\mathrm{i}}$ is called a relevant direction.

(iii) If $\theta_{\mathrm{i}}=0$, we do not know the fate of $z_{\mathrm{i}}$ and we have to go beyond the linear order as explained below; the direction $z_{\mathrm{i}}$ is called marginal in this case.

The notion of relevance or irrelevance is independent of the direction of the flow and of the choice of basis. AS theories correspond to trajectories lying on a critical surface whose tangent space at the fixed point is spanned by the relevant eigenvectors. The number of relevant directions defines the dimension of the critical surface and corresponds to the number of free parameters which have to be fixed by the experiment.

Gauge-Yukawa models in the 211-SCHEME, which are characterized by the set of $\beta$ functions

$$
\begin{aligned}
\partial_{t} \alpha & =\left(-B+C \alpha-\sum_{\mathrm{i}} D_{\mathrm{i}} \alpha_{y_{\mathrm{i}}}\right) \alpha^{2}, \\
\partial_{t} \alpha_{y_{\mathrm{i}}} & =\left(\sum_{j} E_{\mathrm{ij}} \alpha_{y_{\mathrm{j}}}-F_{\mathrm{i}} \alpha\right) \alpha_{y_{\mathrm{i}}},
\end{aligned}
$$

have been recently studied in Refs. [13,15,37]. In the latter equation, the couplings are $\alpha=g^{2} /(4 \pi)^{2}$ and $\alpha_{y_{\mathrm{i}}}=y_{\mathrm{i}}^{2} /(4 \pi)^{2}$, where $g$ is the gauge coupling and $y_{\mathrm{i}}$ are the Yukawa couplings. The quantities $B, E_{\mathrm{ij}}$, and $F_{\mathrm{i}}$ are the one-loop coefficients, while $C$ and $D_{\mathrm{i}}$ are the two-loop ones. It has been show that, depending on the relative sign and magnitude of the coefficients $B, C$, and $C^{\prime}=C-\sum_{\mathrm{ij}} D_{\mathrm{i}}\left(E^{-1}\right)_{\mathrm{ij}} F_{\mathrm{j}}$, this system can have three different types of fixed points [37]:

(i) The Gaussian or noninteracting fixed point, where all couplings are zero.

(ii) The Banks-Zaks fixed point [93], where all the Yukawa couplings vanish.

(iii) The Gauge-Yukawa fixed point, where the gauge and at least one Yukawa coupling are different from zero.

As an example, the phase diagram for the case of a gauge coupling $\alpha$ and a single Yukawa coupling $\alpha_{y}$ with $B>0$ and $C>C^{\prime}>0$ is shown in Fig. 2. In this case, all three kinds of fixed points are present: the Gauge-Yukawa fixed point has both nonvanishing couplings and attracts in the IR trajectories emanating in the UV from both the Gaussian and the Banks-Zaks fixed points.

In the minimal SU(5) GUT model, the system of gauge and top-Yukawa $\beta$ functions takes the form of Eq. (34) with $B>0$ and $C<0$; in this case, no gauge-Yukawa fixed point is present, and the only viable fixed point is the Gaussian one. However, the LVG GUT model considered

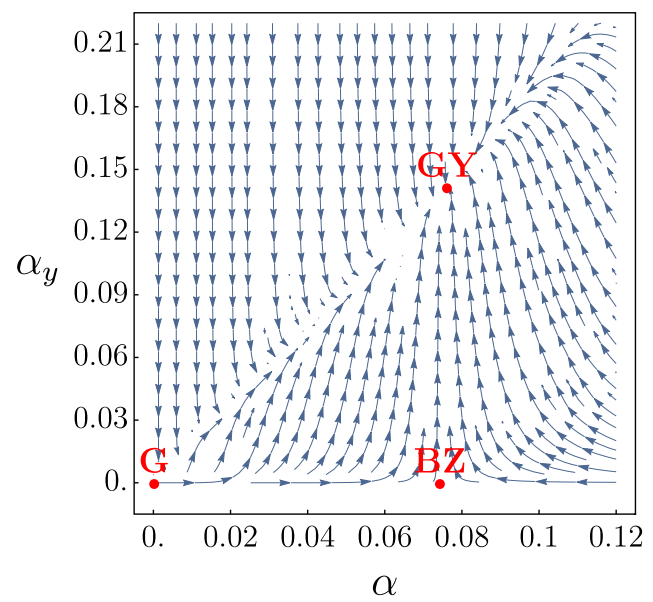

FIG. 2. Example of renormalization group flow in a model with gauge $g$ and Yukawa $y$ couplings. The arrows are pointing toward the IR. There are three fixed points: the Gaussian $(G)$ for vanishing gauge and Yukawa couplings, the Banks-Zaks (BZ) for vanishing Yukawa coupling, and the full interacting GaugeYukawa (GY) fixed point.

in the previous section has additional vectorlike fermions and Yukawa couplings which influence the sign of the coefficients $B, C$, and $C^{\prime}$. This will allow Gauge-Yukawa fixed points to appear, as we will see in the following. Actually, the gauge-Yukawa system of our GUT model, described in Eqs. (16)-(20), is slightly different from the one in Eq. (34) due to nonfactorizable contributions of $\alpha_{\nu}$ to the $\beta$ functions of $\alpha_{5 \Sigma}$ and $\alpha_{24 \Sigma}$. This is indeed the reason why we have considered linear rescaling in the definition of $\alpha$ 's in Eq. (15). Nonetheless, the structure of the gauge $\beta$ function remains the same.

In this work, we compute the fixed points of the full system, including the gauge, Yukawa, and scalar quartic couplings. In general, there are no conditions on the values of the fixed points, and they could take any value. Yet, when we work in perturbation theory, we have to remain within its range of validity. Therefore, we demand that all the couplings have to be sufficiently small at the fixed point. In practice, this means that going to the next order of the perturbative expansion should not appreciably change the position of the fixed point as well as its other properties. This implies that the numerical values of the fixed points must satisfy the conditions

$$
0 \leq \alpha_{5}^{*}<1, \quad\left|\alpha_{\mathrm{t} 10}^{*}\right|<1, \quad\left|\alpha_{z}^{*}\right|<1, \quad\left|\alpha_{X}^{*}\right|<1 .
$$

The complete list of nontrivial fixed points that satisfy these requirements, as a function of $N_{5}$ and $N_{24}$, is shown in Table V. By inspection of Table V, we can see that interacting fixed points can be obtained when $N_{5}=1,2$, 3, 4 and $N_{24}=3$. In this case, the two-loop term of the gauge $\beta$ function turns out to be comparable with the oneloop term as $C \gg B \gtrsim 0$, and the gauge coupling fixed 
TABLE VI. List of the eigenvalues for the fixed points shown in Table V. Negative (positive) eigenvalues correspond to relevant (irrelevant) directions, according to the definitions given in Sec. IV. As for Table V, the bold rows highlight those fixed points which can be matched with the low-energy LVG model at the GUT scale. For the Gaussian fixed point $\mathrm{FP}_{35}$, all couplings are marginal; in particular are marginal and there are no marginally relevant directions.

\begin{tabular}{|c|c|c|c|c|c|c|c|c|c|c|c|c|c|}
\hline & $\left(N_{24}, N_{5}\right)$ & $\theta_{1}$ & $\theta_{2}$ & $\theta_{3}$ & $\theta_{4}$ & $\theta_{5}$ & $\theta_{6}$ & $\theta_{7}$ & $\theta_{8}$ & $\theta_{9}$ & $\theta_{10}$ & & irrel \\
\hline$P_{1}$ & $(3,4)$ & 0.2280 & 0.1662 & 0.1393 & 0.1361 & 0.1020 & 0.06611 & 0.02240 & -0.01197 & -0.01180 & 0.003063 & 2 & 8 \\
\hline $\mathrm{FP}_{2}$ & $(3,4)$ & 0.2291 & 0.1796 & 0.1504 & 0.1361 & 0.1020 & 0.07278 & 0.02240 & -0.01197 & 0.01125 & 0.003063 & 1 & 9 \\
\hline $\mathrm{FP}_{3}$ & $(3,4)$ & 0.2130 & 0.1607 & 0.1376 & 0.1365 & 0.1008 & 0.06133 & 0.02565 & -0.01944 & 0.01428 & 0.003079 & 1 & 9 \\
\hline $\mathrm{FP}_{4}$ & $(3,4)$ & 0.2143 & 0.1836 & 0.1541 & 0.1376 & 0.1008 & 0.07167 & 0.02565 & 0.01802 & 0.01428 & 0.003079 & 0 & 10 \\
\hline $\mathrm{FP}_{5}$ & $(3,3)$ & 0.5034 & 0.3537 & 0.2984 & 0.2958 & 0.2263 & 0.1432 & 0.05686 & -0.04096 & -0.02449 & 0.01311 & 2 & 8 \\
\hline $\mathrm{FP}_{6}$ & $(3,3)$ & 0.5070 & 0.3989 & 0.3348 & 0.2984 & 0.2263 & 0.1662 & 0.05686 & 0.03796 & -0.02449 & 0.01311 & 1 & 9 \\
\hline $\mathrm{FP}_{7}$ & $(3,3)$ & 0.4695 & 0.3439 & 0.3012 & 0.2927 & 0.2230 & 0.1355 & 0.05968 & -0.05031 & 0.03277 & 0.01317 & 1 & 9 \\
\hline $\mathrm{FP}_{8}$ & $(3,3)$ & 0.4729 & 0.4021 & 0.3388 & 0.3012 & 0.2230 & 0.1622 & 0.05968 & 0.04598 & 0.03277 & 0.01317 & 0 & 10 \\
\hline $\mathrm{FP}_{9}$ & $(3,3)$ & -0.3948 & 0.1654 & -0.1357 & -0.1173 & 0.1106 & -0.08280 & 0.05227 & -0.03377 & -0.0 & 09 & 6 & 4 \\
\hline $\mathrm{FP}_{10}$ & $(3,3)$ & -0.3953 & -0.1661 & 0.1654 & -0.1547 & 0.1106 & -0.08280 & -0.04950 & -0.03924 & -0.0 & 0.01009 & 7 & 3 \\
\hline $\mathrm{FP}_{11}$ & $(3,2)$ & 0.8473 & 0.5738 & 0.4971 & 0.4735 & 0.3834 & 0.2371 & 0.1186 & -0.09480 & -0.03685 & 0.03171 & 2 & 8 \\
\hline $\mathrm{FP}_{12}$ & $(3,2)$ & 0.8554 & 0.6748 & 0.5651 & 0.4971 & 0.3834 & 0.2897 & 0.1186 & 0.08533 & -0.03685 & 171 & 1 & 9 \\
\hline $\mathrm{FP}_{13}$ & $(3,2)$ & 0.7901 & 0.5578 & 0.5007 & 0.4720 & 0.3766 & 0.2281 & 0.1152 & -0.1020 & 0.05561 & 0.03185 & 1 & 9 \\
\hline $\mathrm{FP}_{14}$ & $(3,2)$ & 72 & & & 0.5007 & & & & 0.0 & 61 & 35 & 0 & 10 \\
\hline $\mathrm{FP}_{15}$ & $(3,2)$ & -0.2732 & -0.1753 & 0.1670 & -0.1537 & -0.1 & -0.1243 & 41 & -0.08467 & 81 & 84 & 7 & 3 \\
\hline $\mathrm{FP}_{16}$ & $(3,2)$ & -0.2746 & -0.1834 & 0.1670 & -0.1606 & -0.1332 & -0.1243 & -0.1148 & -0.1041 & -0.03738 & 0.02284 & 8 & 2 \\
\hline $\mathrm{FP}_{17}$ & $(3,2)$ & & 0.2690 & 0.2621 & -0.2179 & 0.1752 & -0.1397 & 0.1103 & -0.04398 & 0.02383 & -0.01593 & 4 & 6 \\
\hline $\mathrm{FP}_{18}$ & $(3,2)$ & -0.6248 & 0.2621 & -0.2147 & 0.1752 & 0.1658 & -0.1531 & -0.1397 & -0.06625 & -0.04398 & 0.02383 & 6 & 4 \\
\hline $\mathrm{FP}_{19}$ & $(3,2)$ & -0.6262 & -0.2985 & -0.2795 & 0.2621 & 0.1752 & -0.1521 & -0.1397 & -0.08618 & -0 & 83 & 7 & 3 \\
\hline $\mathrm{FP}_{20}$ & $(3,2)$ & 0.6170 & -0.2776 & 0.2621 & 0.1752 & -0.1425 & -0.1397 & 0.1305 & -0.04398 & -0.0 & 0.02383 & 5 & 5 \\
\hline $\mathrm{FP}_{21}$ & $(3,2)$ & -0.5901 & -0.2688 & 0.2621 & -0.2507 & 0.1752 & -0.1397 & 0.1014 & -0.06413 & -0.04398 & 0.02383 & 6 & 4 \\
\hline $\mathrm{FP}_{22}$ & $(3,2)$ & -0.5953 & 0.2621 & -0.2235 & -0.1920 & 0.1752 & -0.1397 & 0.1382 & -0.04398 & 42 & 383 & 5 & 5 \\
\hline $\mathrm{FP}_{23}$ & $(3,1)$ & -0.4059 & -0.2931 & -0.2557 & 0.2332 & -0.2050 & -0.1736 & -0.1445 & -0.1350 & 0.08109 & 0.04256 & 7 & 3 \\
\hline $\mathrm{FP}_{24}$ & $(3,1)$ & -0.4073 & -0.2989 & -0.2704 & 0.2332 & -0.2075 & -0.2050 & -0.1736 & -0.1350 & -0.08038 & 0.04256 & 8 & 2 \\
\hline $\mathrm{FP}_{25}$ & $(3,1)$ & 0.8658 & 0.4059 & 0.3678 & -0.3052 & 0.2839 & 0.2452 & -0.2155 & -0.05071 & 0.04441 & -0.03803 & 4 & 6 \\
\hline $\mathrm{FP}_{26}$ & $(3,1)$ & -0.8110 & -0.3860 & 0.3678 & -0.3488 & 0.2452 & -0.2155 & 0.1926 & -0.06851 & -0.05071 & 0.04441 & 6 & 4 \\
\hline $\mathrm{FP}_{27}$ & $(3,1)$ & -0.8778 & -0.4429 & -0.4202 & 0.3678 & -0.2917 & 0.2452 & -0.2155 & -0.1541 & -0.05071 & 0.04441 & 7 & 3 \\
\hline $\mathrm{FP}_{28}$ & $(3,1)$ & 0.8697 & -0.4187 & 0.3678 & -0.2838 & 0.2452 & -0.2155 & 0.1384 & -0.09440 & -0.05071 & 0.04441 & 5 & 5 \\
\hline $\mathrm{FP}_{29}$ & $(3,1)$ & -0.8189 & 0.3678 & -0.3411 & -0.2661 & 0.2452 & 0.2409 & -0.2155 & -0.05071 & 0.04806 & 0.04441 & 5 & 5 \\
\hline $\mathrm{FP}_{30}$ & $(3,1)$ & -0.8750 & 0.3678 & 0.3073 & -0.3053 & 0.2452 & -0.2155 & -0.1890 & -0.1044 & -0.05071 & 0.04441 & 6 & 4 \\
\hline $\mathrm{FP}_{31}$ & $(3,1)$ & 1.220 & 0.8271 & 0.7553 & 0.6762 & 0.5844 & 0.3513 & & -0.1951 & 0.07899 & 0.06135 & 1 & 9 \\
\hline $\mathrm{FP}_{32}$ & $(3,1)$ & 1.235 & 1.035 & 0.8625 & 0.7553 & 0.5844 & 0.4547 & 0.2537 & 0.1696 & 0.07899 & 0.06135 & 0 & 10 \\
\hline $\mathrm{FP}_{33}$ & $(3,1)$ & 1.303 & 0.8529 & 0.7524 & 0.6764 & 0.5963 & 0.3596 & 0.2721 & -0.1917 & 0.06115 & -0.04698 & 2 & 8 \\
\hline $\mathrm{FP}_{34}$ & $(3,1)$ & 1.320 & 1.049 & 0.8629 & 0.7524 & 0.5963 & 0.4657 & 0.2721 & 0.1666 & 0.06115 & -0.04698 & 1 & 9 \\
\hline $\mathrm{FP}_{35}$ & $(3, \leq 4)$ & 0 & 0 & $0^{+}$ & $0^{+}$ & $0^{+}$ & $0^{+}$ & $0^{+}$ & 0 & 0 & 0 & 0 & $0^{5}$ \\
\hline
\end{tabular}

point turns out to be much smaller than 1 . The associated critical exponents, which are shown in Table VI, are also much smaller than 1 , and, therefore, we expect the fixed points to be perturbative stable.

\section{RENORMALIZATION GROUP FLOW AND MATCHING WITH THE SM}

In this section, we investigate the existence of asymptotically safe trajectories that emanate from the UV fixed points presented in Table $\mathrm{V}$ and are connected to the SM at the IR scale $\mu=M_{Z}$. As already mentioned, we assume that the vectorlike fermions $\psi_{L}, \psi_{V}$, and $\psi_{G}$-introduced in Sec. II-have a common mass $M_{\mathrm{LVG}}=1 \mathrm{TeV}$, while the vectorlike fermions $\Psi_{5}$ and $\Psi_{24}$-introduced in
Sec. III-have a common mass at the GUT scale. Therefore, we need to consider two matching scales,

$\mu_{1}=M_{\mathrm{LVG}}=1 \mathrm{TeV} \quad$ and $\quad \mu_{2}=M_{\Psi}=M_{\mathrm{GUT}}$,

such that $M_{Z}<M_{\mathrm{LVG}} \ll M_{\Psi}$.

We approximate the decoupling of all vectorlike fermions by considering them as massless above their corresponding matching scale and as infinitely massive below.

The running of the various couplings must be matched at the GUT scale, on the interface between the two models described in Secs. II and III. At this interface, a subtlety regarding the gauge couplings should be taken into account; since their $\beta$ functions have been considered at two-loop order, one-loop matching corrections should 
consequently be accounted for. The latter corrections are given by the expression (cf., for example, Refs. [94,95])

$$
\begin{aligned}
\frac{1}{\alpha_{i}\left(\mu_{2}\right)}= & \frac{1}{\alpha\left(\mu_{2}\right)}-\frac{1}{3}\left[\operatorname{Tr}\left(t_{i, \mathrm{~V}}^{2}\right)-21 \operatorname{Tr}\left(t_{i, \mathrm{~V}}^{2} \log \frac{M_{\mathrm{V}}}{\mu_{2}}\right)\right. \\
& \left.+\operatorname{Tr}\left(t_{i, \mathrm{~S}}^{2} \log \frac{M_{\mathrm{S}}}{\mu_{2}}\right)+8 \operatorname{Tr}\left(t_{i, \mathrm{~F}}^{2} \log \frac{M_{\mathrm{F}}}{\mu_{2}}\right)\right],
\end{aligned}
$$

where $M_{\mathrm{X}}$ with $\mathrm{X}=(\mathrm{V}, \mathrm{S}, \mathrm{F})$ being the mass matrices for the heavy vector bosons [heavy gauge bosons, ghosts, and Goldstone bosons along the broken directions in the adjoint space of $\mathrm{SU}(5)]$, heavy physical scalars, and heavy fermions, respectively. The matrices $t_{i, \mathrm{X}}$ are, instead, the unbroken generators in the representation of the field X, while the trace is performed over the broken subspace of SU(5). In physical terms, the latter Eq. (37) entails that the renormalized gauge couplings $\alpha_{i}$ [after the gauge group $\mathrm{SU}(5)$ is broken down to the SM gauge group] differ from the gauge coupling $\alpha$ of $\mathrm{SU}(5)$ due to the one-loop diagrams where the quantum fluctuations of the heavy particle are integrated out. Numerically, the most important contribution comes from the vector states, if they have a mass different from the matching scale $\mu$. Therefore, we are free to bridge any-reasonably small-mismatch in the running of the gauge couplings by removing the degeneracy in the values of the heavy masses and slightly moving them away from the GUT scale.

The values of the LVG model couplings $\alpha_{i}, \alpha_{\mathrm{t}}, \alpha_{\lambda}$, and $\alpha_{V}$ at $M_{\mathrm{GUT}}$ define the IR target for the LVG GUT model couplings $\alpha_{5}^{2}, \alpha_{\mathrm{t} 10}^{2}, \alpha_{H}$, and $\alpha_{\nu}^{2}$, respectively. In other words, one has to search for those trajectories emanating from the UV fixed points that hit the values of the IR target at the scale $M_{\mathrm{GUT}}$, while flowing down from the fixed points. The other couplings of the LVG GUT model will be consequently determined by this matching condition requirement. Because of the freedom of choosing the mass of the heavy particles $M_{\mathrm{X}}$ slightly different from the matching scale $\mu_{2}$, for any practical purposes, one has to match only the three couplings

$$
\alpha_{\mathrm{t}}\left(\mu_{2}\right)=\alpha_{\mathrm{t} 10}^{2}\left(\mu_{2}\right), \quad \alpha_{\lambda}\left(\mu_{2}\right)=\alpha_{H}\left(\mu_{2}\right), \quad \alpha_{V}\left(\mu_{2}\right)=\alpha_{\nu}^{2}\left(\mu_{2}\right) .
$$

Let us now describe how to obtain such target values. Starting from the scale $M_{Z}$, we first solve the renormalization group flow of the SM up to the first matching scale $\mu_{1}=M_{\mathrm{LVG}}=1 \mathrm{TeV}$. In other words, we integrate the SM $\beta$ functions given in Appendix A with boundary conditions for the SM couplings provided by their experimental values, cf. Table II or Ref. [69]. At the scale $\mu_{1}$, the vectorlike fermions of the LVG model become dynamical such that, from this scale on up to $\mu_{2}=M_{\mathrm{GUT}}$, we integrate the $\beta$ functions in Eqs. (3)-(8), with boundary conditions at $\mu_{1}$ given by the values obtained from the previous integration. Clearly, at the scale $\mu_{1}$, there is one free parameter, namely, the value $\alpha_{V}\left(\mu_{1}\right)$ for the beyond-theSM vectorlike Yukawa coupling. For any values of the latter coupling at $\mu_{1}$, there will be a set of values for $\alpha_{\mathrm{t}}, \alpha_{\lambda}$, and $\alpha_{V}$ at $\mu_{2}$ which defines the IR target for the LVG GUT model. The value $\alpha_{V}\left(\mu_{1}\right)$ has thus to be fine tuned in order for the matching conditions in Eq. (38) to hold, as we are going to explain in the following.

\section{A. Matching procedure}

Given the IR target $\left\{\alpha_{\mathrm{t}}\left(\mu_{2}\right), \alpha_{\lambda}\left(\mu_{2}\right), \alpha_{V}\left(\mu_{2}\right)\right\}$, we have then searched for the existence of those trajectories emanating from the fixed points in Table $\mathrm{V}$ which can be connected to the target itself. To do so, we have integrated the $\beta$ functions for the LVG GUT model given by Eqs. (16)-(25), starting from a point infinitesimally close to the selected fixed point and letting the system to flow down to the IR scale $\mu_{2}=M_{\Psi}=M_{\mathrm{GUT}}$. The initial point of the renormalization group flow is then varied until the trajectory hits, whenever possible, the IR target.

Some comments are in order. The initial point of the RG flow should belong to the UV critical surface in order to guarantee that the flow toward the UV ends at the considered fixed point; say, for example, $\mathrm{FP}_{1}$. This critical surface can be approximated, in the neighborhood of $\mathrm{FP}_{1}$, by its tangent space at $\mathrm{FP}_{1}$, which is defined as the space spanned by the relevant directions at that point. Of course, this approximation is more accurate if the starting point is closer to the fixed point; an infinite numerical precision would be required in order to lie exactly on the critical surface, and a fine-tuning problem is always present while flowing toward the UV. In other words, starting from the IR target, there will usually be a positive RG time $t=\log \left(\mu / \mu_{2}\right)>0$ where the numeric integration breaks down entailing the fact that the RG trajectory is repelled away from the critical surface due to nonzero fluctuations along the irrelevant directions. This is precisely the reason why it is preferable to start the flow in a neighborhood of a fixed point and then flow down to the GUT scale. This guarantees the fact that the physical trajectories are attracted to the critical surface in the IR.

Let us discuss, as a representative case, the fixed point $\mathrm{FP}_{1}$ of Table $\mathrm{V}$ together with the corresponding eigenvalues in Table VI. The tangent space of the critical surface at $\mathrm{FP}_{1}$ is two dimensional and spanned by the eigenvectors $v_{\mathrm{i}}^{(8)}$ and $v_{\mathrm{i}}^{(9)}$ associated, respectively, to the negative eigenvalues $\theta_{8}$ and $\theta_{9}$ of the stability matrix $M_{\mathrm{ij}}$. Any point on this tangent space can thus be parametrized as

$$
\begin{aligned}
\alpha_{\mathrm{i}} & =\alpha_{\mathrm{i}}^{*}+M_{\mathrm{ij}} S_{\mathrm{ik}}\left(\epsilon^{(8)} z_{\mathrm{k}}^{(8)}+\epsilon^{(9)} z_{\mathrm{k}}^{(9)}\right) \\
& =\alpha_{\mathrm{i}}^{*}+M_{\mathrm{ij}} S_{\mathrm{ik}}\left(\epsilon^{(8)} \delta_{\mathrm{k}, 8}+\epsilon^{(9)} \delta_{\mathrm{k}, 9}\right) \\
& =\alpha_{\mathrm{i}}^{*}+\theta_{8} \epsilon^{(8)} v_{\mathrm{i}}^{(8)}+\theta_{9} \epsilon^{(9)} v_{\mathrm{i}}^{(9)} \equiv \alpha_{\mathrm{i}}^{*}+\delta \alpha_{\mathrm{i}},
\end{aligned}
$$


where $\epsilon^{(8)}$ and $\epsilon^{(9)}$ are infinitesimal parameters. In particular, the eigenvector $v_{\mathrm{i}}^{(8)}$ is pointing in the $\alpha_{\mathrm{t} 10}$ direction, whereas $v_{\mathrm{i}}^{(9)}$ induce a displacement along all scalar directions. The freedom of choosing $\epsilon^{(8,9)}$ allow us to match two couplings with the IR target, namely, $\alpha_{\mathrm{t} 10}$ and $\alpha_{H}$.

Let us consider first the behavior of the top-Yukawa coupling. Given a positive, however small, displacement $\delta \alpha_{\mathrm{t} 10}\left(\mu_{0}\right)>0$ at a certain initialization scale $\mu_{0} \gg \mu_{2}$, the top-Yukawa coupling increases while decreasing the energy scale $\mu$, and, eventually, it crosses its IR target value at a scale $\mu_{2}^{\prime}=\mu_{0} \mathrm{e}^{-t^{\prime}}$. On the other hand, given an infinitesimal small, but negative, displacement $\delta \alpha_{H}\left(\mu_{0}\right)<0$, the quartic Higgs self-interaction coupling decreases while decreasing the scale $\mu$, and, eventually, it crosses its IR target value at a scale $\mu_{2}^{\prime \prime}=\mu_{0} \mathrm{e}^{-t^{\prime \prime}}$. Usually, the two scales $\mu_{2}^{\prime}$ and $\mu_{2}^{\prime \prime}$ are different. Nevertheless, fixing $\epsilon^{(9)}$ while varying $\epsilon^{(8)}$ (or vice versa), it is possible to fine-tune the initial conditions $\delta \alpha_{\mathrm{t}}\left(\mu_{0}\right)$ and $\delta \alpha_{H}\left(\mu_{0}\right)$ such that $\mu_{2}^{\prime}=\mu_{2}^{\prime \prime}$ to any arbitrarily chosen numerical precision. Since the initial scale $\mu_{0}$ is not a priori fixed, it is legit to impose $\mu_{2}^{\prime}=$ $\mu_{2}^{\prime \prime}=\mu_{2}$ such that the fine-tuned initial conditions $\delta \alpha_{\mathrm{t} 10}\left(\mu_{0}\right)$ and $\delta \alpha_{H}\left(\mu_{0}\right)$ correspond to the correct values at the scale $\mu_{0}=\mu_{2} \mathrm{e}^{t^{\prime}}=\mu_{2} \mathrm{e}^{t^{\prime \prime}}$ required in order to match $\alpha_{\mathrm{t} 10}$ and $\alpha_{H}$ to their IR values in Eq. (38).

There is a technical difficulty regarding the latter finetuning procedure. The RG flow for the quartic Higgs selfinteraction $\alpha_{H}(\mu)$ is such that close to the energy scale $\mu_{2}^{\prime \prime}$ it varies very fast due to the presence of an IR singularity below, yet very close to, the scale $\mu_{2}^{\prime \prime}$. At this singular point, the quartic Higgs self-interaction diverges to infinitely negative values. In other words, $\alpha_{H}(\mu)$ remains very much close to its fixed point value until the energy scale approaches $\mu_{2}^{\prime \prime}$ and $\alpha_{H}(\mu)$ starts to decreases very fast toward the singularity. It is in this running toward the singularity that the RG trajectory of $\alpha_{H}(\mu)$ is intercepted and stopped at exactly the scale $\mu_{2}^{\prime \prime}$ where $\alpha_{H}$ equals the (negative) value of the IR target. Because of the condition $\mu_{2}^{\prime \prime}=\mu_{2}\left(=\mu_{2}^{\prime}\right)$, we can claim that this singularity does not encode a physical inconsistency in our LVG model because it occurs below the GUT scale where, indeed, a different system of differential equations holds. The only drawback of the presence of this (alleged) singularity below the GUT scale is that, in order to satisfy the equality $\mu_{2}^{\prime \prime}=\mu_{2}=\mu_{2}^{\prime}$, a high degree of fine-tuning for the initial conditions $\alpha_{\mathrm{t} 10}\left(\mu_{0}\right)$ and $\alpha_{H}\left(\mu_{0}\right)$ is required. In other words, by an appropriate choice for the initial conditions on the (tangent space of the) critical surface at the $\mu_{0}$ scale, it is possible to move the singularity of $\alpha_{H}$ below the GUT scale. This singularity thus becomes physically not worrisome, as below the GUT scale another system is considered.

Subsequently, one has to read off the value $\alpha_{\nu}^{2}\left(\mu_{2}\right)$, which usually does not coincide with $\alpha_{V}\left(\mu_{2}\right)$. To match this last condition, we have exploited the freedom of choosing a different boundary condition for $\alpha_{V}\left(\mu_{1}\right)$. Varying this latter value, one falls into one of these cases $\alpha_{\nu}^{2}\left(\mu_{2}\right) \gtrless \alpha_{V}\left(\mu_{2}\right)$ such that a simple bisection algorithm allows one to fine tune the condition $\alpha_{\nu}^{2}\left(\mu_{2}\right)=\alpha_{V}\left(\mu_{2}\right)$ to any arbitrary chosen numerical precision. Notice that for any different value of $\alpha_{V}\left(\mu_{1}\right)$, the above procedure of fine-tuning the couplings $\alpha_{\mathrm{t} 10}$ and $\alpha_{H}$ has to be repeated, increasing the numerical effort required to satisfy all conditions in Eq. (38).



FIG. 3. The renormalization group flow for the LVG model approaching the fixed point $\mathrm{FP}_{1}$ in the UV limit $\mu \rightarrow \infty$. Below the GUT scale (at about $10^{16} \mathrm{GeV}$ and highlighted by the vertical line in the middle), the gauge couplings $\alpha_{i}$, the top-Yukawa couplings $\alpha_{\mathrm{t}}$, and the Higgs quartic self-interaction are represented with boundary conditions given at the $M_{Z}$ scale. The vectorlike fermion fields $\psi_{L}, \psi_{V}$, and $\psi_{G}$, with multiplicity $N_{\mathrm{f}}=1$, enter in the dynamics at the energy scale of $1 \mathrm{TeV}$ (highlighted by the gray vertical line on the left). The value for the beyond-the-SM Yukawa coupling $\alpha_{V}$ at $1 \mathrm{TeV}$ is a free parameter to be fine tuned in order to satisfy the matching conditions in Eq. (38). Above the GUT scale, the SU(5) gauge group is restored, and further vectorlike fermion fields are included; in particular, there are three flavors of $\Psi_{24}$ and four flavors of $\Psi_{5}$. The gauge coupling $\alpha_{5}^{2}$, the top-Yukawa coupling $\alpha_{\mathrm{t} 10}^{2}$, the Higgs coupling $\alpha_{H}$, and the vectorlike Yukawa coupling $\alpha_{\nu}^{2}$ are represented. 
TABLE VII. For the fixed point $\mathrm{FP}_{1}$, we report the variation of the predicted value for the beyond-the-SM Yukawa coupling $\alpha_{V}$ with respect to the initialization scale $M_{\mathrm{LVG}}=\mu_{1}$ of the vectorlike fermions. We illustrate also the variation of the gauge, topYukawa, and Higgs quartic self-interaction couplings at the GUT scale $\mu_{2}$.

\begin{tabular}{lcccc}
\hline \hline$M_{\mathrm{LVG}}(\mathrm{TeV})$ & $\alpha_{V}\left(\mu_{1}\right)$ & $\alpha_{\mathrm{GUT}}\left(\mu_{2}\right)$ & $\alpha_{t}\left(\mu_{2}\right)$ & $\alpha_{\lambda}\left(\mu_{2}\right)$ \\
\hline 0.1 & 0.0009767 & 0.002322 & 0.001238 & -0.001970 \\
1 & 0.0009317 & 0.002322 & 0.001396 & -0.002055 \\
10 & 0.0008927 & 0.002322 & 0.001497 & -0.002044 \\
100 & 0.0008572 & 0.002323 & 0.001562 & -0.001987 \\
1000 & 0.0008239 & 0.002323 & 0.001603 & -0.001904 \\
\hline \hline
\end{tabular}

Once all three conditions in Eq. (38) have been satisfied, we can finally plot the full RG flow from the $M_{Z}$ scale up to the considered fixed point. As an example, we present how the fixed point $\mathrm{FP}_{1}$ can be connected with the physics at the scale $M_{Z}$ in Fig. 3. At the interface between the two models, that is at the GUT scale, two RG flows described by two sets of first-order differential equations have to be matched. Because of this first-order structure, the RG trajectories are only required to be continuous; however, no constraint on the first derivatives, namely, the values of the $\beta$ functions at the GUT scale, should be imposed. This is particularly evident in the RG trajectory of the Higgs self-interaction coupling. The small discontinuity in the gauge couplings is due to the finite correction induced by the one-loop matching condition given by Eq. (37). Let us emphasize the fact that the low-energy value for the beyond-the-SM Yukawa coupling $\alpha_{V}$ at the scale $M_{\mathrm{LVG}}=1 \mathrm{TeV}$ represents a physical prediction. This prediction is the result of the matching conditions at the interface between the two systems below and above the GUT scale.

In Sec. II A, see in particular Table III, we have seen that there is a certain freedom in choosing the energy scale from which the vectorlike fermions of the LVG model become dynamical. It is therefore interesting to check whether this freedom is preserved also by the matching procedure or the latter imposes some sort of bounds on the possible values of $M_{\mathrm{LVG}}$. As a testing case, we have again chosen the fixed point $\mathrm{FP}_{1}$, and in Table VII, we report the corresponding predictions for the coupling $\alpha_{V}$ at different $M_{\mathrm{LVG}}$ scales. We conclude that there are no further restrictions on the $\mu_{1}$ scale and that the matching procedure can be successfully carried for a broad range of such scale. We expect the same behavior also for the other fixed points which allow for a matching between the two models at the interface scale $\mu_{2}$.

The above method can be applied to any fixed point in Table V. Quite in general, the latter Eq. (39) can be written as

$$
\alpha_{\mathrm{i}}=\alpha_{\mathrm{i}}^{*}+\sum_{\theta_{a}<0} \epsilon^{(a)} v_{\mathrm{i}}^{(a)}
$$

where the sum is over all the relevant eigendirections associated to negative eigenvalues and $\epsilon^{(a)}$ are all free parameters. It seems that the degree of difficulty increases with the number of relevant directions, i.e., the number of free parameters to be fixed. Nevertheless, we have found that, in order to verify whether a fixed point can be connected with a physical trajectory to the physics at the GUT scale, it is always possible to reduce the number of $\epsilon^{(a)}$ to be fixed to the minimal value of 2 . These two parameters are always associated to those directionseventually different from $v_{\mathrm{i}}^{(8,9)}$ depending on the fixed point -which result in the same RG behavior for the couplings $\alpha_{\mathrm{t} 10}$ and $\alpha_{H}$ as described above.

In Tables $\mathrm{V}$ and VI, we have highlighted in bold all possible fixed points which possess, at least, one physical trajectory which hits the IR target $\left\{\alpha_{\mathrm{t}}\left(\mu_{2}\right), \alpha_{\lambda}\left(\mu_{2}\right), \alpha_{V}\left(\mu_{2}\right)\right\}$ at the GUT scale satisfying the matching conditions in Eq. (38). To understand why the only highlighted fixed points can be matched with the IR target at the GUT scale, it can be useful to visualize in which directions the linear eigenperturbations drive the RG flow. To this aim, in Table VIII, we have listed with the symbol $\mathbf{a}$ all those couplings which get perturbed by a nonzero infinitesimal displacement $\epsilon^{(a)}$ along all relevant directions. It is clear that all fixed points which allow for a match with the IR target at $\mu_{2}$ share the same feature: both top-Yukawa

TABLE VIII. Schematic representation of those couplings which are perturbed away from the fixed point values by a nonzero infinitesimal displacement $\epsilon^{(a)}$ along all relevant $\left(\theta_{a}<0\right)$ directions $v^{(a)}$ spanning the tangent space of the critical surface at the corresponding fixed point.

\begin{tabular}{|c|c|c|c|c|c|c|c|}
\hline & $\left(N_{24}, N_{5}\right)$ & $\delta \alpha_{5}$ & $\delta \alpha_{\mathrm{t} 10}$ & $\delta \alpha_{24 \Sigma}$ & $\delta \alpha_{5 \Sigma}$ & $\delta \alpha_{\nu}$ & $\delta \alpha_{X}$ \\
\hline $\mathrm{FP}_{1}$ & $(3,4)$ & - & - & - & - & - & - \\
\hline $\mathrm{FP}_{2}$ & $(3,4)$ & - & घ & - & - & - & - \\
\hline $\mathrm{FP}_{3}$ & $(3,4)$ & - & - & - & - & - & - \\
\hline $\mathrm{FP}_{4}$ & $(3,4)$ & - & - & - & - & - & - \\
\hline $\mathrm{FP}_{5}$ & $(3,3)$ & - & - & - & - & - & - \\
\hline $\mathrm{FP}_{6}$ & $(3,3)$ & - & - & - & - & - & - \\
\hline $\mathrm{FP}_{7}$ & $(3,3)$ & - & - & - & - & - & a \\
\hline $\mathrm{FP}_{8}$ & $(3,3)$ & - & - & - & - & - & - \\
\hline $\mathrm{FP}_{9,10}$ & $(3,3)$ & - & - & - & - & - & - \\
\hline $\mathrm{FP}_{11}$ & $(3,2)$ & - & - & - & - & - & - \\
\hline $\mathrm{FP}_{12}$ & $(3,2)$ & - & - & - & - & - & - \\
\hline $\mathrm{FP}_{13}$ & $(3,2)$ & - & - & - & - & - & - \\
\hline $\mathrm{FP}_{14}$ & $(3,2)$ & - & - & - & - & - & - \\
\hline $\mathrm{FP}_{15,16}$ & $(3,2)$ & - & - & - & - & - & - \\
\hline $\mathrm{FP}_{17-22}$ & $(3,2)$ & - & - & - & - & - & - \\
\hline $\mathrm{FP}_{23,24}$ & $(3,1)$ & - & - & - & - & - & ! \\
\hline $\mathrm{FP}_{25-30}$ & $(3,1)$ & - & - & - & - & - & ! \\
\hline $\mathrm{FP}_{31}$ & $(3,1)$ & - & - & - & - & - & ! \\
\hline $\mathrm{FP}_{32}$ & $(3,1)$ & - & - & - & - & - & - \\
\hline $\mathrm{FP}_{33}$ & $(3,1)$ & - & - & - & - & - & - \\
\hline $\mathrm{FP}_{34}$ & $(3,1)$ & - & - & - & - & - & - \\
\hline
\end{tabular}


and quartic scalar self-interaction couplings have to be perturbed away from their fixed point values. All other fixed points cannot be matched with the LVG model at the GUT scale because either only the top-Yukawa or the scalar couplings (or neither of them) are perturbed by moving away from the fixed point along the critical surface.

Predictivity in the low-energy regime is an important feature in any AS theories. All relevant directions at a certain fixed point (i.e., with negative eigenvalues) are associated to free parameters of the theory. On the contrary, couplings associated to irrelevant directions are predicted in the IR. Table VIII shows that not all the fixed points that allow for a matching at the GUT scale have the same degree of predictivity. For example, for the fixed point $\mathrm{FP}_{1}-\mathrm{as}$ well as for $\mathrm{FP}_{5,11,33}$ - the coupling $\alpha_{\nu}$ in the low-energy regime represents a prediction. This is not the case for the fixed points $\mathrm{FP}_{15,16,23,24}$ where $\alpha_{\nu}$ represents, instead, an additional free parameter in the theory.

\section{B. Gaussian fixed point}

We have also investigated the Gaussian fixed point which is present for all values of $N_{5}$ and $N_{24}$. If we further demand the non-Abelian gauge coupling $\alpha_{5}$ to be asymptotically free (AF), then the multiplicities of the vectorlike fermions are restricted to be

$N_{24}=3, N_{5} \leq 4, \quad N_{24}=2, N_{5} \leq 9 \quad$ or

$N_{24}=1, N_{5} \leq 14$.

Given a model with an AF non-Abelian gauge sector, one can try to investigate whether the AF gauge coupling can drive all other couplings toward the Gaussian fixed point. To address this question, one can study the quasi-fixed-points [96-99] (also called in the literature fixed flows [32] or eigenvalue conditions $[100,101])$ for the rescaled couplings

$$
\hat{\alpha}_{X}=\frac{\alpha_{X}}{\alpha_{5}^{2}} \quad \text { and } \quad \hat{\alpha}_{z}=\frac{\alpha_{z}}{\alpha_{5}},
$$

such that any finite quasi-fixed-point $\left(\hat{\alpha}_{X}^{*}, \hat{\alpha}_{z}^{*}\right)$ represents a specific trajectory along which the UV behavior of the scalar couplings $\alpha_{X}$ and the Yukawa couplings $\alpha_{z}$ is locked to follow the AF gauge coupling $\alpha_{5}$.

Among all possible AF scenarios for $\alpha_{5}$ given in Eq. (41), we have found the existence of quasi-fixed-points $\left(\hat{\alpha}_{X}^{*}, \hat{\alpha}_{z}^{*}\right)$ only for the combinations

$$
N_{24}=3, \quad N_{5}=(1,2,4) .
$$

Notice that, for example, the minimal configuration where only one $\Psi_{5}$ and $\Psi_{24}$ are present above the GUT scale does not have quasi-fixed-points in the Yukawa-scalar sector. It appears that higher multiplicities are required in order for the gauge coupling $\alpha_{5}$ to drive all other couplings toward the Gaussian fixed point. Yet, among these possibilities, we have found that none of the corresponding quasi-fixed- points provides viable trajectories which can be matched with the IR target $\left\{\alpha_{\mathrm{t}}\left(\mu_{2}\right), \alpha_{\lambda}\left(\mu_{2}\right), \alpha_{V}\left(\mu_{2}\right)\right\}$ at the GUT scale. In other words, within our LVG GUT model, we have found that no total $\mathrm{AF}$ trajectories can be found, thus rendering the interacting fixed points which allow for a matching at the GUT scale even more special.

The vectorlike fermion content corresponding to $N_{24}=3, N_{5}=3$ has been discussed in Ref. [32], in which the authors presented the existence of quasi-fixed-points and argued that realistic total AF GUT models can be constructed. The reason for this disagreement might come from the fact that our Yukawa sector is simpler than the one considered in Ref. [32]. For example, we do not consider the Yukawa interaction term among the vectorlike fermion $\Psi_{5}$ and the SM GUT field $\chi_{\overline{5}}$ (exchanging an adjoint scalar $\Sigma$ ), which is precisely the Yukawa coupling (together with the top Yukawa) acquiring a nontrivial quasi-fixed-point. In the light of these observations regarding the Gaussian fixed point, it would be interesting to study how the interacting fixed points presented in Table $\mathrm{V}$ change after including these terms, in particular those for which a matching with the SM at the GUT scale is possible.

\section{ANOTHER MINIMAL EXTENSION: THE 2U2Q MODEL}

The work of Ref. [34] classifies all minimal SM extension with vectorlike fermions at the $\mathrm{TeV}$ scale in which good coupling unification is obtained. Among those, another relevant ${ }^{2}$ choice is the $2 U 2 Q$ model that represents a "fourth-generation" scenario. In the case of the 2U2Q model, the $Q$ and $U$ labels stand for fields that are vectorlike fermion multiplets transforming, respectively, under the $(\mathbf{3}, \mathbf{2}, 1 / 6)$ and $(\mathbf{3}, 1,2 / 3)$ representations of the SM, in analogy to the SM quark doublets and up singlets. The $2 \mathrm{U} 2 \mathrm{Q}$ model corresponds to adding to the SM two $\psi_{Q}$ and two $\psi_{U}$ multiplets at the $\mathrm{TeV}$ scale, with quantum numbers shown in Table IX. The Lagrangian of the $2 \mathrm{U} 2 \mathrm{Q}$ model then reads

$$
\begin{aligned}
\mathcal{L}_{2 \mathrm{U} 2 \mathrm{Q}}= & \mathcal{L}_{\mathrm{SM}}+\bar{\psi}_{Q}^{I} \mathrm{i} \not D \psi_{Q}^{I}+\bar{\psi}_{U}^{J} \mathrm{i} \not \supset \psi_{U}^{J}-M_{Q} \bar{\psi}_{Q}^{I} \psi_{Q}^{I} \\
& -M_{U} \bar{\psi}_{U}^{J} \psi_{U}^{J}-y_{Q} \sum_{I, J} \bar{\psi}_{Q}^{I} \psi_{U}^{J} H^{c}+\text { H.c. }
\end{aligned}
$$

where $I, J=(1,2)$ and $H^{c}=\mathrm{i} \sigma_{2} H^{*}$ is the charge conjugated Higgs field. $y_{Q}$ is the coupling of the new Yukawa interaction involving the Higgs and the vectorlike fermions. This term breaks the flavor symmetry to a diagonal subgroup $\mathrm{SU}(2)_{Q} \times \mathrm{SU}(2)_{U} \rightarrow \mathrm{SU}(2)_{D}$, where the $Q$ and $U$ vectorlike fermions rotate with the same transformation. At the unification scale, the vectorlike fermions of the $2 \mathrm{U} 2 \mathrm{Q}$ model in Table IX are embedded into proper multiples of SU(5); we assume that the fields $\psi_{Q}$ and $\psi_{U}$ get embedded into

\footnotetext{
${ }^{2}$ Also, the $(U+D)$ model of Ref. [34] has promising properties below the GUT scale, and it has been studied in Ref. [35].
} 
TABLE IX. Quantum numbers and multiplicities of the vectorlike fermions in the $2 \mathrm{U} 2 \mathrm{Q}$ model.

\begin{tabular}{lcccc}
\hline \hline Fields & $\mathrm{SU}(3)_{\mathrm{c}}$ & $\mathrm{SU}(2)_{\mathrm{L}}$ & $\mathrm{U}(1)_{\mathrm{Y}}$ & $N_{\mathrm{f}}$ \\
\hline$\psi_{Q}$ & $\mathbf{3}$ & $\mathbf{2}$ & $1 / 6$ & 2 \\
$\psi_{U}$ & $\mathbf{3}$ & 1 & $2 / 3$ & 2 \\
\hline \hline
\end{tabular}

TABLE X. Quantum numbers and multiplicities of the vectorlike fermions in the $2 \mathrm{U} 2 \mathrm{Q}$ GUT model.

\begin{tabular}{lcc}
\hline \hline Fields & $\mathrm{SU}(5)$ & $N_{\mathrm{f}}$ \\
\hline$\Psi_{10}$ & $\mathbf{1 0}$ & $N_{10}$ \\
\hline \hline
\end{tabular}

vectorlike fermions fields $\Psi_{10}$, with multiplicity $N_{10} \geq 2$, transforming under the antisymmetric representation ${ }^{3}$ of SU(5), see Table X. The Lagrangian of the 2U2Q GUT model is

$$
\begin{aligned}
\mathcal{L}_{2 \mathrm{U} 2 \mathrm{Q}}^{\mathrm{GUT}}= & \mathcal{L}_{\mathrm{SM}}^{\mathrm{GUT}}+\frac{1}{2} \operatorname{Tr}\left(\bar{\Psi}_{10} \mathrm{i} \not \supset \Psi_{10}\right)-\frac{1}{2} M_{10} \operatorname{Tr}\left(\bar{\Psi}_{10} \Psi_{10}\right) \\
& +\frac{y_{10}}{8} \sum_{I, J} \varepsilon_{5} \bar{\Psi}_{10}^{c I} \Psi_{10}^{J} \Phi+\text { H.c. } \\
& +y_{10}^{\prime} \sum_{I, J} \operatorname{Tr}\left(\bar{\Psi}_{10}^{I} \Sigma \Psi_{10}^{J}\right)
\end{aligned}
$$

where $I, J=\left(1,2, \ldots, N_{10}\right)$ are the flavor indices for the $\Psi_{10}$ field. Let us notice that not all possible SU(5) invariant Yukawa terms have been considered with the given matter field content. For the sake of simplicity, we have not considered, for example, Yukawa interactions between $\Psi_{10}$ and $\chi_{10}$ or $\chi_{5}$. Therefore, no Yukawa interactions between the SM fermions and the extra vectorlike fermions are retained.

Can this choice of vectorlike fermions give rise to an asymptotically safe SU(5) GUT model? The answer is no. We computed the $\beta$ functions of this model and studied its fixed points. We found that, for any value of $N_{10} \geq 2$, the only viable fixed point is the Gaussian one; yet, no good matching with the SM at low energy is possible. For completeness, we give the $\beta$ functions of the 2U2Q model in Appendix D.

\section{CONCLUSIONS}

The goal of having an asymptotically safe extension of the Standard Model is a powerful motivation in searching for physics beyond the SM. It provides a guiding principle that still remains viable after others, like supersymmetry or compositeness, are waning.

The simplest way to turn the Landau pole of the SM into a fixed point is through a GUT scenario. The addition of

\footnotetext{
${ }^{3}$ The antisymmetric representation of $\mathrm{SU}(5)$ can be decomposed under the SM gauge group as follows [87]: $\mathbf{1 0}=(\mathbf{3}, \mathbf{2}, 1 / 6) \oplus(\overline{\mathbf{3}}, 1,-2 / 3) \oplus(1,1,1)$.
}

few vectorlike fermions at the $1 \mathrm{TeV}$ scale makes an $\mathrm{SU}(5)$ GUT unification of the SM gauge couplings possible and consistent with all experimental constraints. We consider what we dubbed the LVG model, which contains the same features of a split SUSY scenario, and the 2U2Q model, which has the features of a fourth-generation scenario.

For the LVG model, we found a GUT embedding that has a fixed point for the gauge coupling that is interacting - as opposed to the more familiar case of the asymptotically free limit common to all unbroken non-Aabelian gauge models. We find this an interesting feature. It could perhaps be tested in cosmology, for instance, in physics around and above the GUT phase transition. We also check the 2U2Q model, but no matching to the SM can be found in this case.

We are aware that the LVG model as it stands cannot yet be considered completely satisfactory.

For one thing, the known problem of the stability of the Higgs potential remains, as it does in the SM. The scale at which the potential crosses to negative values for the coefficient of the quartic term is about the same as in the $\mathrm{SM}$, as computed at the one-loop order. It is known $[5,7,8]$ that this value increases as higher-loop orders are computed and included, and we expect the same to happen in the case of the LVF model. The model is metastable, but the time scale for its decay is longer than the age of the Universe.

Moreover, fine-tuning is required in order to split the masses of scalars and fermions belonging to the same $\mathrm{SU}(5)$ representations as we go to low energy. This is an outstanding problem of all GUT models-indeed, the very motivation for the original naturalness requirement [102-104] — for which we have not attempted a solution.

These shortcomings notwithstanding, the asymptotically safe extension of the SM model represented by the LVG model is noteworthy. The theory is UV complete. All the couplings of the model are perturbative and remain so along the entire renormalization group flow up and beyond the Planck scale. The existence of such a model is highly nontrivial as shown by the lack of fixed points matchable to the SM for models without unification [18] or other choices of the vectorlike fermion content- to wit, the $2 \mathrm{U} 2 \mathrm{Q}$ model.

\section{ACKNOWLEDGMENTS}

This work was supported in part by the Natural Sciences and Engineering Research Council of Canada (NSERC). We thank R. Percacci and R. Sondenheimer for valuable discussions. M. F. is affiliated to the Physics Department of the University of Trieste, the Scuola Internazionale Superiore di Studi Avanzati (SISSA), and the Institute for Fundamental Physics of the Universe (IFPU), Trieste, Italy, the support of which is acknowledged. A. T. and A. U. would like to thank Associazione di Fondazioni e Casse di Risparmi Spa and INFN for financial support through the Young Investigator Training Program 2018. A. U. acknowledges support by the DFG under Grants No. 398579334 (Gi328/9-1) and No. 396639009 
(ZA 958/2-1). C. M. N. is supported by the VIE-UIS Postdoctoral Fellowship Program No. 2021000126.

\section{APPENDIX A: THE SM $\beta$ FUNCTIONS IN THE 211-SCHEME}

In the 211-SCHEME, the $\beta$ functions of the SM gauge couplings are computed at two loops, while the $\beta$ functions of the top-Yukawa and Higgs quartic coupling are computed at one loop. They are given by (see, for example, Ref. [105])

$$
\begin{gathered}
\beta_{1}^{\mathrm{SM}, \mathrm{NLO}}=\left(\frac{41}{3}+\frac{199}{9} \alpha_{1}+9 \alpha_{2}+\frac{88}{3} \alpha_{3}-\frac{17}{3} \alpha_{\mathrm{t}}\right) \alpha_{1}^{2}, \\
\beta_{1}^{\mathrm{SM}, \mathrm{NLO}}=\left(-\frac{19}{3}+3 \alpha_{1}+\frac{35}{3} \alpha_{2}+24 \alpha_{3}-3 \alpha_{\mathrm{t}}\right) \alpha_{2}^{2}, \\
\beta_{1}^{\mathrm{SM}, \mathrm{NLO}}=\left(-14+\frac{11}{3} \alpha_{1}+9 \alpha_{2}-52 \alpha_{3}-4 \alpha_{\mathrm{t}}\right) \alpha_{3}^{2}, \\
\beta_{\mathrm{t}}^{\mathrm{SM}, \mathrm{LO}}=\left(-\frac{17}{6} \alpha_{1}-\frac{9}{2} \alpha_{2}-16 \alpha_{3}+9 \alpha_{\mathrm{t}}\right) \alpha_{\mathrm{t}},
\end{gathered}
$$

$$
\begin{aligned}
\beta_{\lambda}^{\mathrm{SM}, \mathrm{LO}}= & 12 \alpha_{\lambda}^{2}-3\left(\alpha_{1}+3 \alpha_{2}-4 \alpha_{\mathrm{t}}\right) \alpha_{\lambda} \\
& +\frac{3}{4}\left(\alpha_{1}^{2}+2 \alpha_{1} \alpha_{2}+3 \alpha_{2}^{2}\right)-12 \alpha_{\mathrm{t}}^{2}
\end{aligned}
$$

\section{APPENDIX B: VECTORLIKE FERMIONS CONTRIBUTIONS TO THE SM $\beta$ FUNCTIONS}

Consider Dirac vectorlike fermions $\psi$, with multiplicity $N_{\mathrm{f}}$, that belong to the representation $R_{3}$ of $\mathrm{SU}(3)_{\mathrm{c}}$ and $R_{2}$ of $\mathrm{SU}(2)_{\mathrm{L}}$ and have hypercharge Y. The one- and two-loop contributions to the running of the SM gauge couplings are given by [106-109]

$$
\begin{aligned}
& \beta_{1}^{\mathrm{NLO}}=\frac{1}{2}\left(B_{1}+M_{1} \alpha_{1}+H_{1} \alpha_{2}+G_{1} \alpha_{3}\right) \alpha_{1}, \\
& \beta_{2}^{\mathrm{NLO}}=\frac{1}{2}\left(B_{2}+M_{2} \alpha_{1}+H_{2} \alpha_{2}+G_{2} \alpha_{3}\right) \alpha_{2}, \\
& \beta_{3}^{\mathrm{NLO}}=\frac{1}{2}\left(B_{3}+M_{3} \alpha_{1}+H_{3} \alpha_{2}+G_{3} \alpha_{3}\right) \alpha_{3},
\end{aligned}
$$

where

$$
\begin{gathered}
B_{1}=\frac{8}{3} N_{\mathrm{f}} \mathrm{Y}^{2} d_{R_{2}} d_{R_{3}}, \quad B_{2}=\frac{8}{3} N_{\mathrm{f}} S_{R_{2}} d_{R_{3}}, \quad B_{3}=\frac{8}{3} N_{\mathrm{f}} S_{R_{3}} d_{R_{2}}, \\
M_{1}=8 \mathrm{Y}^{4} N_{\mathrm{f}} d_{R_{2}} d_{R_{3}}, \quad M_{2}=4 N_{\mathrm{f}} S_{R_{2}} d_{R_{3}}\left(2 C_{R_{2}}+\frac{20}{3}\right),
\end{gathered}
$$

The Casimir invariants $C_{R_{i}}$ and Dynkin indices $S_{R_{i}}$ are defined in general as

$$
\begin{gathered}
d_{R_{2}}=2 \ell+1, \quad d_{R_{3}}=\frac{1}{2}(p+1)(q+1)(p+q+2), \\
C_{R_{2}}=\ell(\ell+1), \quad C_{R_{3}}=p+q+\frac{1}{3}\left(p^{2}+q^{2}+p q\right), \\
S_{R_{2}}=\frac{1}{3} d_{R_{2}} C_{R_{2}}, \quad S_{R_{3}}=\frac{1}{8} d_{R_{3}} C_{R_{3}},
\end{gathered}
$$

where $\ell=0, \frac{1}{2}, 1, \frac{3}{2}, \ldots$ denotes the highest weight of $R_{2}$ and $(p, q)$ (with $\left.p, q=0,1,2 \ldots\right)$ denotes the weights of $R_{3}$. For our specific LVG model, we have

$$
\psi_{L}: \ell=\frac{1}{2}, p=q=0, \quad \psi_{V}: \ell=1, p=q=0, \quad \psi_{G}: \ell=0, p=q=1 .
$$


The contributions to the gauge, top-Yukawa, and Higgs quartic couplings coming from the vectorlike fermion Yukawa coupling $\alpha_{V}$, as well as the contribution to the $\beta$ function $\partial_{t} \alpha_{V}$ itself, have been computed using the results in Ref. [68]. Let us consider two Dirac vectorlike fermions $\psi_{1}$ and $\psi_{2}$ in two, generically different, representations of the SM gauge group, such that a gauge-invariant Yukawa interaction term can be constructed. In case $\psi_{1}$ and $\psi_{2}$ have multiplicity $N_{1}$ and $N_{2}$, respectively, then this interaction term takes the form of

$$
-y_{I J} \bar{\psi}_{1}^{I} \psi_{2}^{J} H+\text { H.c. }
$$

where $y_{I J}$ is a complex $N_{1} \times N_{2}$ matrix. For generic representations of $\psi_{1}$ and $\psi_{2}$, we were not able to provide general formulas for the contributions of $y_{I J}$ to the $\beta$ functions for the gauge couplings $\alpha_{i}$, the top-Yukawa coupling $\alpha_{\mathrm{t}}$, or the Higgs quartic self-interaction. For this reason, an explicit computation of these contributions seems unavoidable.

Let us therefore consider our specific case where $\psi_{1}=\psi_{L}$ and $\psi_{2}=\psi_{V}$. We can slightly generalize the vectorlike fermion Yukawa interaction in Eq. (1) by keeping arbitrary the multiplicities $N_{1}$ and $N_{2}$ and assuming that all vectorlike fermions interact among each other with the same Yukawa coupling $y_{V}$. The contribution to the gauge couplings enters as a two-loop diagram where inside a fermionic loop the Higgs field is exchanged. This contribution is given by (cf. Eq. (31) in Ref. [68])

$$
\begin{aligned}
\partial_{t} \alpha_{i}= & \ldots-\frac{2 \alpha_{i}}{d\left(\mathcal{G}_{i}\right)}\left[C_{R_{i}}^{(L)}\left(2 d_{R_{2}}^{(L)}\right) d_{R_{3}}^{(L)} \mathcal{Y}_{2 \mathrm{~F}}^{(L)}\right. \\
& \left.+C_{R_{i}}^{(V)} d_{R_{2}}^{(V)} d_{R_{3}}^{(V)} \mathcal{Y}_{2 \mathrm{~F}}^{(V)}\right] N_{1} N_{2},
\end{aligned}
$$

where $d\left(\mathcal{G}_{i}\right)$ is the dimension of the gauge group $\mathcal{G}_{i}$ and the superscripts $(L)$ and $(V)$ refer to the vectorlike fermions $\psi_{L}$ and $\psi_{V}$, respectively. For $i=1$, the Casimir invariants simply reduce to the square of the hypercharges. The extra factor 2 for the $L$ representation is due to the fact that $\psi_{L}$ is a Diraclike field while $\psi_{V}$ is a Majorana-like field. The coefficients $\mathcal{Y}_{2 \mathrm{~F}}$ are the (real) eigenvalues of the matrix product $\mathcal{Y}^{a} \mathcal{Y}^{a \dagger}$, where $\mathcal{Y}^{a}$ is the symmetrized matrix of the Yukawa interaction couplings between all Weyl components of the vectorlike fermionic fields and the real scalar component $\phi^{a}$ of $H$ (the construction of the matrices $\mathcal{Y}^{a}$ will be clarified later in Appendix C). For our specific case, we have that

$$
\mathcal{Y}_{2 \mathrm{~F}}^{(L)}=3 \alpha_{V}^{2}, \quad \mathcal{Y}_{2 \mathrm{~F}}^{(V)}=4 \alpha_{V}^{2} .
$$

Clearly, the contribution to the $\beta$ function of the strong gauge coupling is zero since both $\psi_{L}$ and $\psi_{V}$ are singlet under the $\mathrm{SU}(3)_{\mathrm{c}}$ gauge group.

The contribution to the top-Yukawa $\beta$ function comes from the scalar anomalous dimension which has an extra contribution due to the exchange of the vector like fermions. This extra term reads (cf. Eq. (33) in Ref. [68])

$$
\partial_{t} \alpha_{\mathrm{t}}=\beta_{\mathrm{t}}^{\mathrm{SM}, \mathrm{LO}}+2 \alpha_{\mathrm{t}} \mathcal{Y}_{2 \mathrm{~S}} N_{1} N_{2}
$$

where $\mathcal{Y}_{2 \mathrm{~S}}$ is the eigenvalue of the scalar loop matrix $\frac{1}{2} \operatorname{Tr}\left(\mathcal{Y}^{a^{\dagger}} \mathcal{Y}^{b}+\mathcal{Y}^{b \dagger} \mathcal{Y}^{a}\right)=\mathcal{Y}_{2 S} \delta^{a b}$. For our specific case, $\mathcal{Y}_{2 S}=$ $6 \alpha_{V}$. This justifies also the linear contribution in $\alpha_{V}$ to the $\beta$ function for the Higgs quartic coupling, which is $4 \alpha_{\lambda} \mathcal{Y}_{2 S}$.

Similarly, the top-Yukawa contribution to $\partial_{t} \alpha_{V}$ comes from the Higgs anomalous dimension where the top quark is exchanged. In the latter case, the top contribution to the scalar anomalous dimension is $\mathcal{Y}_{2 \mathrm{~S}}^{\text {top }}=3 \alpha_{\mathrm{t}}$, where the factor 3 comes from the color structure. The gauge contributions to the $\beta$ function for the vectorlike Yukawa coupling can be written in terms of the Casimir invariants for the two vectorlike representations, such that we can write (cf. Eq. (33) in Ref. [68])

$$
\partial_{t} \alpha_{V}=15 N_{1} N_{2} \alpha_{V}^{2}+2 \alpha_{V} \mathcal{Y}_{2 \mathrm{~S}}^{\text {top }}-6 \alpha_{i}\left[C_{R_{i}}^{(L)}+C_{R_{i}}^{(V)}\right] \alpha_{V},
$$

where the quadratic contribution in $\alpha_{V}$ depends on the particular form of the matrices $\mathcal{Y}^{a}$ and is the sum of contributions coming from the fermion anomalous dimension and the renormalization of the operator in Eq. (B13).

Regarding the $\beta$ function for the quartic Higgs coupling, the linear term in $\alpha_{V}$ is due to the scalar anomalous dimension, and the quadratic contribution in $\alpha_{V}$ depends again on the particular form of the Yukawa matrices $\mathcal{Y}^{a}$. We thus obtain (cf. Eq. (38) in Ref. [68])

$$
\partial_{t} \alpha_{\lambda}=\beta_{\lambda}^{\mathrm{SM}, \mathrm{LO}}+4 N_{1} N_{2} \mathcal{Y}_{2 \mathrm{~S}} \alpha_{\lambda}-48 N_{1}^{2} N_{2}^{2} \alpha_{V}^{2} .
$$

\section{APPENDIX C: The $\beta$ FUNCTIONS FOR THE SU(5) LVG MODEL}

The gauge and scalar quartic contributions to the $\beta$ functions of the scalar potential in Eq. (13) have been computed at one loop in Ref. [110]. Given the definitions in Eq. (15) and defining $\bar{\beta}_{X}=\partial_{t} \alpha_{X}$, we have

$$
\begin{gathered}
\bar{\beta}_{\Sigma}=\frac{32}{5} \alpha_{\Sigma}^{2}+\alpha_{\Sigma}\left(12 \alpha_{\Sigma}^{\prime}-60 \alpha_{5}^{2}\right)+8 \alpha_{H \Sigma}^{\prime 2}+30 \alpha_{5}^{4}, \\
\bar{\beta}_{\Sigma}^{\prime}=32 \alpha_{\Sigma}^{\prime 2}+\alpha_{\Sigma}^{\prime}\left(\frac{94}{5} \alpha_{\Sigma}-60 \alpha_{5}^{2}\right)+16 \alpha_{H \Sigma} \alpha_{H \Sigma}^{\prime}+40 \alpha_{H \Sigma}^{2}+\frac{84}{25} \alpha_{\Sigma}^{2}+18 \alpha_{5}^{4},
\end{gathered}
$$




$$
\begin{gathered}
\bar{\beta}_{H}=18 \alpha_{H}^{2}-\frac{144}{5} \alpha_{H} \alpha_{5}^{2}+96 \alpha_{H \Sigma}^{2}+\frac{192}{5} \alpha_{H \Sigma} \alpha_{H \Sigma}^{\prime}+\frac{264}{25} \alpha_{H \Sigma}^{2}+\frac{198}{25} \alpha_{5}^{4}, \\
\bar{\beta}_{H \Sigma}=8 \alpha_{H \Sigma}^{2}+\alpha_{H \Sigma}\left(12 \alpha_{H}+\frac{47}{5} \alpha_{\Sigma}+26 \alpha_{\Sigma}^{\prime}-\frac{222}{5} \alpha_{5}^{2}\right)+\alpha_{H \Sigma}^{\prime}\left(2 \alpha_{H}+2 \alpha_{H \Sigma}^{\prime}+\frac{28}{25} \alpha_{\Sigma}+\frac{24}{5} \alpha_{\Sigma}^{\prime}\right)+\frac{3}{2} \alpha_{5}^{4}, \\
\bar{\beta}_{H \Sigma}^{\prime}=\frac{42}{5} \alpha_{H \Sigma}^{\prime 2}+\alpha_{H \Sigma}^{\prime}\left(16 \alpha_{H \Sigma}+\frac{19}{5} \alpha_{\Sigma}+2 \alpha_{\Sigma}^{\prime}+2 \alpha_{H}-\frac{222}{5} \alpha_{5}^{2}\right)+\frac{15}{2} \alpha_{5}^{4} .
\end{gathered}
$$

\section{SU(5) scalar potential}

To compute the extra contributions to the latter $\beta$ functions due to the presence of the vectorlike fermion Yukawa couplings $\alpha_{5 \Sigma}, \alpha_{24 \Sigma}$, and $\alpha_{\nu}$, we have made use of the general results of Ref. [68] [cf. Eqs. (38)-(43) therein]. Following the notation of Ref. [68], we have first expanded the scalar fields of our model, namely, $\Phi(x)$ and $\Sigma(x)$, in terms of their real scalar components $\phi_{a}$. In other words,

$$
\Phi(x)=\frac{1}{\sqrt{2}}\left(\phi_{1}(x)+\mathrm{i} \phi_{6}(x), \ldots, \phi_{5}(x)+\mathrm{i} \phi_{10}(x)\right)^{\mathrm{T}}, \quad \Sigma(x)=\sum_{A=1}^{24} \phi_{10+A}(x) T^{A},
$$

where $T^{A}$ are the generators of the SU(5) gauge group, normalized in such a way that $\operatorname{Tr}\left(T^{A} T^{B}\right)=\frac{1}{2} \delta^{A B}$. The quartic scalar potential in Eq. (13) can thus be brought into the form

$$
V^{\text {quartic }}(\Phi, \Sigma)=\frac{1}{4 !} \lambda_{a b c d} \phi_{a} \phi_{b} \phi_{c} \phi_{d}, \quad \lambda_{a b c d}=\left.\frac{4 !}{\mathrm{P}[a, b, c, d]} V\right|_{[a, b, c, d]},
$$

where $\lambda_{a b c d}$ is a total symmetric rank-4 tensor ${ }^{4}$ whose entries contain the scalar interactions $\lambda_{X} .\left.V\right|_{[a, b, c, d]}$ is the coefficient in front of the quartic operator $\phi_{a} \phi_{b} \phi_{c} \phi_{d}$, and $\mathrm{P}[a, b, c, d]$ is the number of nonequivalent permutations of the set of indices $\{a, b, c, d\}$. Similarly, all the fermionic fields, namely, $\chi_{5}, \chi_{10}, \Psi_{5}$, and $\Psi_{24}$, have to be expanded in terms of their Weyl lefthanded two-component spinors $\psi_{i}$. For an explicit example, let us consider the vectorlike fermion representations

$$
\begin{gathered}
\Psi_{5}=\left(\xi_{1}, \ldots, \xi_{5}\right)^{\mathrm{T}}, \quad \xi_{i}=\xi_{i, \mathrm{~L}}+\xi_{i, \mathrm{R}} \equiv \psi_{i}-\mathrm{i} \sigma_{2} \psi_{i+5}^{*}, \\
\Psi_{24}=\sum_{A=1}^{24} \xi_{5+A} T^{A}, \quad \xi_{5+A} \equiv \psi_{10+A}-\mathrm{i} \sigma_{2} \psi_{10+A}^{*},
\end{gathered}
$$

where all the right-handed Weyl components are expressed as the charged conjugation of some Weyl left-handed spinors (notice that the vectorlike fermion $\Psi_{24}$ is a Majorana-like fermion).

Given the above decomposition, the Yukawa interaction terms in Eqs. (12) and (14) can thus be written as

$$
\mathcal{L}^{\text {Yukawa }}=-\frac{1}{2} \mathcal{Y}_{i j}^{a} \psi_{i} \zeta \psi_{j} \phi_{a}+\text { H.c. }
$$

where $\zeta= \pm \mathrm{i} \sigma_{2}$ and $\mathcal{Y}^{a}$ are symmetric Yukawa matrices.

The extra contributions in Eqs. (21)-(25) can be obtained from Eqs. (40) and (41) in Ref. [68]. In particular, the contributions quadratic in $\alpha_{z}$ come from the scalars anomalous dimensions and are proportional

\footnotetext{
${ }^{4}$ Generally speaking, for a total symmetric tensor, i.e., symmetric in all its indices, of rank $r$ where all indices can assume $n$ different values, the number of independent components is given exactly by the number of combinations with repetition $C_{r}^{n}=(n+r-1) ! /(r !(n-1) !)$. In our specific case, $\lambda_{a b c d}$ has $C_{4}^{36}=66045$ independent components.
}

to the eigenvalues of the scalar loop matrix $\frac{1}{2} \operatorname{Tr}\left(\mathcal{Y}^{a^{\dagger}} \mathcal{Y}^{b}+\mathcal{Y}^{b \dagger} \mathcal{Y}^{a}\right)$. These eigenvalues are, for the present model,

$\mathcal{Y}_{2 \mathrm{~S}}^{H}=3 \alpha_{\mathrm{t} 10}^{2}+\frac{24}{5} N_{5} N_{24} \alpha_{\nu}^{2}, \quad \mathcal{Y}_{2 \mathrm{~S}}^{\Sigma}=\frac{21}{20} N_{24} \alpha_{24 \Sigma}^{2}+N_{5} \alpha_{5 \Sigma}^{2}$.

The quartic contributions in $\alpha_{z}$ are instead due to a fermionic loop where four fermions are exchanged among the four scalar fields. This contribution is obtained from

$$
\frac{\partial_{t} \lambda_{a b c d}}{(4 \pi)^{2}}=\ldots-\sum \operatorname{Tr}\left[\mathcal{Y}^{a} \mathcal{Y}^{b \dagger} \mathcal{Y}^{c} \mathcal{Y}^{d^{\dagger}}\right]
$$

where the sum is over all 4 ! permutations of the indices $\{a, b, c, d\}$.

\section{Gauge-Yukawa subsystem of the SU(5) LVG model}

The $\beta$ functions for the SU(5) gauge coupling as well as for the Yukawa couplings have also been computed by 
mean of the general formulas for a generic gauged quantum field theory given in Ref. [68].

In particular, the $\beta$ functions for the Yukawa couplings have been computed from Eq. (33) of Ref. [68]. The extra contributions to $\partial_{t} \alpha_{\mathrm{t} 10}$ due to the vectorlike Yukawa interaction $\alpha_{\nu}$, as well as the top contribution to $\partial_{t} \alpha_{\nu}$, come from the Higgs anomalous dimension, cf. Eq. (C11). The terms proportional to $N_{5}$ and $N_{24}$ in Eqs. (18) and (19) come, instead, from the anomalous dimension of the $\Sigma$ scalar field, cf. again Eq. (C11). The other Yukawa contributions are a nontrivial sum of the fermionic anomalous dimensions and the renormalization of the operator $\mathcal{Y}_{i j}^{a} \psi_{i} \zeta \psi_{j}$. Therefore, an explicit computation of the first two terms in Eq. (33) of Ref. [68] is required.

The gauge contributions to the $\beta$ functions of the Yukawa couplings are obtained by computing the Casimir invariants for the different fermionic representations. For a generic $\mathrm{SU}(N)$ gauge group, the Casimir for the fundamental, antisymmetric, and adjoint representations are, respectively,

$$
C_{N}=\frac{N^{2}-1}{2 N}, \quad C_{\text {antisymm }}=\frac{(N+1)(N-2)}{N}, \quad C_{\text {adj }}=N,
$$

such that the gauge contributions to the Yukawa $\beta$ functions for our LVG SU(5) GUT model are

$$
\begin{array}{ll}
\partial_{t} \alpha_{\mathrm{t} 10}=\ldots-3 \alpha_{5}^{2}\left(C_{\mathbf{1 0}}+C_{\mathbf{1 0}}\right) \alpha_{\mathrm{t} 10}, & \partial_{t} \alpha_{5 \Sigma}=\ldots-3 \alpha_{5}^{2}\left(C_{\mathbf{5}}+C_{\mathbf{5}}\right) \alpha_{5 \Sigma}, \\
\partial_{t} \alpha_{24 \Sigma}=\ldots-3 \alpha_{5}^{2}\left(C_{\mathbf{2 4}}+C_{\mathbf{2 4}}\right) \alpha_{24 \Sigma}, & \partial_{t} \alpha_{\nu}=\ldots-3 \alpha_{5}^{2}\left(C_{\mathbf{5}}+C_{\mathbf{2 4}}\right) \alpha_{\nu},
\end{array}
$$

where the sum in parentheses refers to the sum over the fermions which are exchanged in the one-loop diagrams where a gauge boson is exchanged.

The two-loop $\beta$ function for the SU(5) gauge coupling has been computed from Eq. (30) of Ref. [68], where the terms proportional to the square of the Yukawa couplings can be written as

$$
\partial_{t} \alpha_{5}=\ldots-\frac{\alpha_{5}^{3}}{d(\mathcal{G})}\left[C_{\mathbf{1 0}} d_{\mathbf{1 0}} \mathcal{Y}_{2 \mathrm{~F}}\left(\chi_{10}\right)+C_{\mathbf{5}} d_{\mathbf{5}} \mathcal{Y}_{2 \mathrm{~F}}\left(\Psi_{5}\right)\left(2 N_{5}\right)+C_{\mathbf{2 4}} d_{\mathbf{2 4}} \mathcal{Y}_{2 \mathrm{~F}}\left(\Psi_{24}\right) N_{24}\right]
$$

where $d(\mathcal{G})=24$ is the dimension of the $\mathrm{SU}(5)$ gauge group and $d_{\mathrm{F}_{i}}$, with $F_{i}=(\mathbf{5}, \mathbf{1 0}, \mathbf{2 4})$, is the dimension of the different fermionic representations. Let us notice the presence of an extra factor of 2 in the multiplicity of $\Psi_{5}$ due to the fact that it is a Dirac-like fermion. The eigenvalues of the matrix product $\mathcal{Y}^{a} \mathcal{Y}^{a \dagger}$ are

$$
\mathcal{Y}_{2 \mathrm{~F}}\left(\chi_{10}\right)=3 \alpha_{\mathrm{t} 10}^{2}, \quad \mathcal{Y}_{2 \mathrm{~F}}\left(\Psi_{5}\right)=\frac{12}{5} \alpha_{5 \Sigma}^{2}+\frac{12}{5} N_{24} \alpha_{\nu}^{2}, \quad \mathcal{Y}_{2 \mathrm{~F}}\left(\Psi_{24}\right)=\frac{21}{20} \alpha_{24 \Sigma}^{2}+N_{5} \alpha_{\nu}^{2}
$$

The one-loop contributions to the RG flow of $\alpha_{5}$ are obtained by first computing the Dynkin indices for the different (fermionic and scalar) representations. Generally speaking, given a representation $R$ of a gauge group $\mathcal{G}$, we have

$$
S_{R}=\frac{d_{R} C_{R}}{d(\mathcal{G})}
$$

such that for $\mathrm{SU}(N)$ we obtain

$S_{N}=\frac{1}{2}, \quad S_{\text {antisymm }}=\frac{N-2}{2}, \quad S_{\text {adj }}=N$.

For our specific SU(5) GUT model, we thus have

$\partial_{t} \alpha_{5}=-\alpha_{5}^{3}\left[\frac{11}{3} C(\mathcal{G})-\sum_{i}\left(\frac{2}{3} S_{\mathrm{F}_{i}}+\frac{1}{6} S_{\mathrm{S}_{i}}\right)\right]+\beta_{\alpha_{5}}^{\mathrm{NLO}}$, where the Casimir for the $\mathrm{SU}(5)$ gauge group is $C(\mathcal{G})=5$ and the sum is over all the fermionic and scalar representations. Each generation of the SM fermionic sector can be fitted in the representations $\overline{\mathbf{5}}$ and $\mathbf{1 0}$. On the other hand, the scalar sector of the SM GUT theory is composed of a complex fundamental representation and a real adjoint representation. Taking into account also the vectorlike fermion representations, we finally have

$$
\begin{aligned}
\partial_{t} \alpha_{5}= & -\alpha_{5}^{3}\left\{\frac{11}{3} C(\mathcal{G})-\frac{2}{3}\left[S_{\mathrm{F}}\left(\chi_{\overline{5}}\right)+S_{\mathrm{F}}\left(\chi_{10}\right)\right] N_{\mathrm{g}}\right. \\
& -\frac{2}{3}\left[2 N_{5} S_{\mathrm{F}}\left(\Psi_{5}\right)+N_{24} S_{\mathrm{F}}\left(\Psi_{24}\right)\right] \\
& \left.-\frac{1}{6}\left[2 S_{\mathrm{S}}(\Phi)+S_{\mathrm{S}}(\Sigma)\right]\right\}+\beta_{\alpha_{5}}^{\mathrm{NLO}}
\end{aligned}
$$

where $N_{\mathrm{g}}=3$ is the generation number and the extra factor of 2 for the contributions of $\Phi$ and $\Psi_{5}$ come from the fact 
that $\Psi_{5}$ is a Dirac-like fermion and $\Phi$ is composed of two real 5-plets, respectively, the real and imaginary parts. Substituting the values in Eq. (C19) into the latter equation, we obtain the one-loop contribution of Eq. (16). Given the Casimir and Dynkin indices for the different representations, it is straightforward to obtain the two-loop contribution in Eq. (16) from Eq. (30) of Ref. [68].

\section{APPENDIX D: THE $\beta$ FUNCTIONS FOR THE 2U2Q MODEL}

Using the definitions in Eq. (2) and the following rescaling for the coupling $\alpha_{Q}$,

$$
\alpha_{Q}=\frac{y_{Q}^{2}}{(4 \pi)^{2}},
$$

the $\beta$ functions of the $2 \mathrm{U} 2 \mathrm{Q}$ model read

$$
\partial_{t} \alpha_{1}=\beta_{1}^{\mathrm{SM}, \mathrm{NLO}}+\left(8+\frac{86}{9} \alpha_{1}+2 \alpha_{2}+32 \alpha_{3}-\frac{136}{3} \alpha_{Q}\right) \alpha_{1}^{2},
$$

$$
\partial_{t} \alpha_{2}=\beta_{2}^{\mathrm{SM}, \mathrm{NLO}}+\left(8+\frac{2}{3} \alpha_{1}+98 \alpha_{2}+32 \alpha_{3}-24 \alpha_{Q}\right) \alpha_{2}^{2},
$$

$$
\partial_{t} \alpha_{3}=\beta_{3}^{\mathrm{SM}, \mathrm{NLO}}+\left(8+4 \alpha_{1}+12 \alpha_{2}+152 \alpha_{3}-32 \alpha_{Q}\right) \alpha_{3}^{2},
$$

$$
\begin{gathered}
\partial_{t} \alpha_{\mathrm{t}}=\beta_{\mathrm{t}}^{\mathrm{SM}, \mathrm{LO}}+48 \alpha_{Q} \alpha_{\mathrm{t}}, \\
\partial_{t} \alpha_{Q}=\left(-\frac{17}{6} \alpha_{1}-\frac{9}{2} \alpha_{2}-16 \alpha_{3}+60 \alpha_{Q}+6 \alpha_{\mathrm{t}}\right) \alpha_{Q}, \\
\partial_{t} \alpha_{\lambda}=\beta_{\lambda}^{\mathrm{SM}, \mathrm{LO}}+96 \alpha_{Q} \alpha_{\lambda}-384 \alpha_{Q}^{2},
\end{gathered}
$$

where $\beta_{i}^{\mathrm{SM}, \mathrm{NLO}}, \beta_{\mathrm{t}}^{\mathrm{SM}, \mathrm{LO}}$, and $\beta_{\lambda}^{\mathrm{SM}, \mathrm{LO}}$ are the $\mathrm{SM} \beta$ functions previously given in Appendix A. The new terms arising from the presence of the extra vectorlike fermions $\psi_{U}$ and $\psi_{Q}$ are explicitly shown. Their contributions to the running of the gauge couplings have been computed using the formulas in Appendix B. The extra contributions due to the vectorlike Yukawa coupling $\alpha_{Q}$ as well as the $\beta$ function of $\alpha_{Q}$ itself have been computed using the results of Ref. [68] [cf. Eqs. (30), (33), and (38) therein].

In the following, we present the $\beta$ functions of the 2U2Q GUT model where the vectorlike fermions $\psi_{U}$ and $\psi_{Q}$ are embedded into the antisymmetric representation of SU(5). Let us denote $y_{10}$ and $y_{10}^{\prime}$ the two Yukawa couplings of the vectorlike fermions with the scalar fields $\Phi$ and $\Sigma$, respectively. We define

$$
\alpha_{10}=\frac{y_{10}}{4 \pi}, \quad \alpha_{10}^{\prime}=\frac{y_{10}^{\prime}}{4 \pi},
$$

together with the definitions given in Eq. (15) for the gauge, top-Yukawa, and scalar couplings. For the sake of simplicity, we assume that the latter Yukawa interactions are diagonal in the flavor indices $I, J=\left(1,2, \ldots, N_{10}\right)$, such that different flavors of the matter field $\Psi_{10}$ do not interact among each others. The sum in Eq. (45) reduces to $\sum_{I}(\ldots)$. The RG flow equations for the gauge-Yukawa subsystem are

$$
\begin{gathered}
\partial_{t} \alpha_{5}=-\frac{40-6 N_{10}}{3} \alpha_{5}^{3}-\frac{1184-1074 N_{10}}{15} \alpha_{5}^{5} \\
-\left(\frac{9}{2} \alpha_{\mathrm{t} 10}^{2}+9 N_{10} \alpha_{10}^{2}+\frac{54}{5} N_{10} \alpha_{10}^{\prime 2}\right) \alpha_{5}^{3}, \\
\partial_{t} \alpha_{\mathrm{t} 10}=\left(6 \alpha_{\mathrm{t} 10}^{2}+6 N_{10} \alpha_{10}^{2}-\frac{108}{5} \alpha_{5}^{2}\right) \alpha_{\mathrm{t} 10}, \\
\partial_{t} \alpha_{10}=\left[3\left(1+2 N_{10}\right) \alpha_{10}^{2}+3 \alpha_{\mathrm{t} 10}^{2}-\frac{6}{5} \alpha_{10}^{\prime 2}-\frac{108}{5} \alpha_{5}^{2}\right] \alpha_{10}, \\
\partial_{t} \alpha_{10}^{\prime}=\left[\frac{29+15 N_{10}}{5} \alpha_{10}^{\prime 2}-\alpha_{10}^{2}-\frac{108}{5} \alpha_{5}^{2}\right] \alpha_{10}^{\prime} .
\end{gathered}
$$

We conclude the set of $\beta$ functions by listing the running of the scalar couplings.

$$
\begin{gathered}
\partial_{t} \alpha_{\Sigma}=\bar{\beta}_{\Sigma}+12 N_{10}\left(\alpha_{\Sigma}+\alpha_{10}^{\prime 2}\right) \alpha_{10}^{\prime 2}, \\
\partial_{t} \alpha_{\Sigma}^{\prime}=\bar{\beta}_{\Sigma}^{\prime}+12 N_{10}\left(\alpha_{\Sigma}^{\prime}-\alpha_{10}^{\prime 2}\right) \alpha_{10}^{\prime 2}, \\
\partial_{t} \alpha_{H}=\bar{\beta}_{H}+\left(12 \alpha_{\mathrm{t} 10}^{2}+12 N_{10} \alpha_{10}^{2}\right) \alpha_{H}-12 \alpha_{\mathrm{t}}^{4}-24 N_{10} \alpha_{10}^{4}, \\
\partial_{t} \alpha_{H \Sigma}=\bar{\beta}_{H \Sigma}+\left(6 \alpha_{\mathrm{t} 10}^{2}+12 N_{10} \alpha_{10}^{2}+6 N_{10} \alpha_{10}^{\prime 2}\right) \alpha_{H \Sigma} \\
-4 N_{10} \alpha_{10}^{2} \alpha_{10}^{\prime 2}, \\
\partial_{t} \alpha_{H \Sigma}^{\prime}=\bar{\beta}_{H \Sigma}^{\prime}+\left(6 \alpha_{\mathrm{t} 10}^{2}+12 N_{10} \alpha_{10}^{2}+6 N_{10} \alpha_{10}^{\prime 2}\right) \alpha_{H \Sigma}^{\prime} \\
-4 N_{10} \alpha_{10}^{2} \alpha_{10}^{\prime 2},
\end{gathered}
$$

where the pure gauge and scalar contributions $\bar{\beta}_{\Sigma}, \bar{\beta}_{\Sigma}^{\prime}, \bar{\beta}_{H}$, $\bar{\beta}_{H \Sigma}$, and $\bar{\beta}_{H \Sigma}^{\prime}$ are given in Appendix C. 
[1] K. G. Wilson and J. B. Kogut, The renormalization group and the epsilon expansion, Phys. Rep. 12, 75 (1974).

[2] S. Weinberg, Ultraviolet divergences in quantum theories of gravitation, in General Relativity: An Einstein Centenary Survey (Cambridge University press, Cambridge, 1980), pp. 790-831.

[3] D. J. Gross and F. Wilczek, Ultraviolet Behavior of Nonabelian Gauge Theories, Phys. Rev. Lett. 30, 1343 (1973).

[4] H. D. Politzer, Reliable Perturbative Results for Strong Interactions?, Phys. Rev. Lett. 30, 1346 (1973).

[5] G. Isidori, G. Ridolfi, and A. Strumia, On the metastability of the standard model vacuum, Nucl. Phys. B609, 387 (2001).

[6] J. Ellis, J. R. Espinosa, G. F. Giudice, A. Hoecker, and A. Riotto, The probable fate of the standard model, Phys. Lett. B 679, 369 (2009).

[7] J. Elias-Miro, J. R. Espinosa, G. F. Giudice, G. Isidori, A. Riotto, and A. Strumia, Higgs mass implications on the stability of the electroweak vacuum, Phys. Lett. B 709, 222 (2012).

[8] G. Degrassi, S. Di Vita, J. Elias-Miro, J. R. Espinosa, G. F. Giudice, G. Isidori, and A. Strumia, Higgs mass and vacuum stability in the standard model at NNLO, J. High Energy Phys. 08 (2012) 098.

[9] S. Alekhin, A. Djouadi, and S. Moch, The top quark and Higgs boson masses and the stability of the electroweak vacuum, Phys. Lett. B 716, 214 (2012).

[10] I. Masina, Higgs boson and top quark masses as tests of electroweak vacuum stability, Phys. Rev. D 87, 053001 (2013).

[11] D. Buttazzo, G. Degrassi, P. P. Giardino, G. F. Giudice, F. Sala, A. Salvio, and A. Strumia, Investigating the nearcriticality of the Higgs boson, J. High Energy Phys. 12 (2013) 089.

[12] D. F. Litim and F. Sannino, Asymptotic safety guaranteed, J. High Energy Phys. 12 (2014) 178.

[13] A. D. Bond and D.F. Litim, More asymptotic safety guaranteed, Phys. Rev. D 97, 085008 (2018).

[14] B. Holdom, J. Ren, and C. Zhang, Stable asymptotically free extensions (SAFEs) of the standard model, J. High Energy Phys. 03 (2015) 028.

[15] A. D. Bond, G. Hiller, K. Kowalska, and D. F. Litim, Directions for model building from asymptotic safety, J. High Energy Phys. 08 (2017) 004.

[16] R. Mann, J. Meffe, F. Sannino, T. Steele, Z.-W. Wang, and C. Zhang, Asymptotically Safe Standard Model via Vectorlike Fermions, Phys. Rev. Lett. 119, 261802 (2017).

[17] G. M. Pelaggi, A. D. Plascencia, A. Salvio, F. Sannino, J. Smirnov, and A. Strumia, Asymptotically safe Standard Model extensions?, Phys. Rev. D 97, 095013 (2018).

[18] D. Barducci, M. Fabbrichesi, C. M. Nieto, R. Percacci, and V. Skrinjar, In search of a UV completion of the standard model-378,000 models that don't work, J. High Energy Phys. 11 (2018) 057.

[19] G. Hiller, C. Hormigos-Feliu, D. F. Litim, and T. Steudtner, Model building from asymptotic safety with Higgs and flavor portals, Phys. Rev. D 102, 095023 (2020).

[20] H. Gies and J. Ziebell, Asymptotically safe QED, Eur. Phys. J. C 80, 607 (2020).
[21] A. Eichhorn, H. Gies, J. Jaeckel, T. Plehn, M. M. Scherer, and R. Sondenheimer, The Higgs mass and the scale of new physics, J. High Energy Phys. 04 (2015) 022.

[22] R. Alkofer, A. Eichhorn, A. Held, C. M. Nieto, R. Percacci, and M. Schröfl, Quark masses and mixings in minimally parameterized UV completions of the Standard Model, Ann. Phys. (Amsterdam) 421, 168282 (2020).

[23] H. Georgi and S. L. Glashow, Unity of All Elementary Particle Forces, Phys. Rev. Lett. 32, 438 (1974).

[24] J. C. Pati and A. Salam, Lepton number as the fourth color, Phys. Rev. D 10, 275 (1974); , Erratum, Phys. Rev.D 11, 703 (1975).

[25] R. N. Mohapatra and J. C. Pati, Left-right gauge symmetry and an "isoconjugate" model of CP violation, Phys. Rev. D 11, 566 (1975).

[26] H. Fritzsch and P. Minkowski, Unified interactions of leptons and hadrons, Ann. Phys. (N.Y.) 93, 193 (1975).

[27] H. Georgi, The state of the art-gauge theories, AIP Conf. Proc. 23, 575 (1975).

[28] B. Bajc and F. Sannino, Asymptotically safe grand unification, J. High Energy Phys. 12 (2016) 141.

[29] E. Molinaro, F. Sannino, and Z. Wang, Asymptotically safe Pati-Salam theory, Phys. Rev. D 98, 115007 (2018).

[30] Z.-W. Wang, A. Al Balushi, R. Mann, and H.-M. Jiang, Safe trinification, Phys. Rev. D 99, 115017 (2019).

[31] F. Sannino, J. Smirnov, and Z.-W. Wang, Asymptotically safe clockwork mechanism, Phys. Rev. D 100, 075009 (2019).

[32] G. F. Giudice, G. Isidori, A. Salvio, and A. Strumia, Softened gravity and the extension of the standard model up to infinite energy, J. High Energy Phys. 02 (2015) 137.

[33] G. M. Pelaggi, A. Strumia, and S. Vignali, Totally asymptotically free trinification, J. High Energy Phys. 08 (2015) 130.

[34] G. F. Giudice and A. Romanino, Split supersymmetry, Nucl. Phys. B699, 65 (2004); , Erratum, Nucl. Phys. B706, 487 (2005).

[35] B. Bhattacherjee, P. Byakti, A. Kushwaha, and S. K. Vempati, Unification with Vector-like fermions and signals at LHC, J. High Energy Phys. 05 (2018) 090.

[36] K. Kowalska and D. Kumar, Road map through the desert: Unification with vector-like fermions, J. High Energy Phys. 12 (2019) 094.

[37] A. D. Bond and D. F. Litim, Theorems for asymptotic safety of gauge theories, Eur. Phys. J. C 77, 429 (2017); , Erratum, Eur. Phys. J. C 77, 525 (2017).

[38] K. Abe et al., Search for proton decay via $p \rightarrow e^{+} \pi^{0}$ and $p \rightarrow \mu^{+} \pi^{0}$ in 0.31 megaton per years exposure of the Super-Kamiokande water Cherenkov detector, Phys. Rev. D 95, 012004 (2017).

[39] T. G. Rizzo, Desert grand unified theories and new light degrees of freedom, Phys. Rev. D 45, R3903 (1992).

[40] Z. Bin and Z. Han-Qing, Top quark, heavy fermions and the composite Higgs boson, Commun. Theor. Phys. 35, 162 (2001).

[41] D. Choudhury, T. M. P. Tait, and C. E. M. Wagner, Beautiful mirrors and precision electroweak data, Phys. Rev. D 65, 053002 (2002). 
[42] L.-F. LI and F. WU, Coupling constant unification in extensions of standard model, Int. J. Mod. Phys. A 19, 3217 (2004).

[43] D. E. Morrissey and C. E. M. Wagner, Beautiful mirrors, unification of couplings, and collider phenomenology, Phys. Rev. D 69, 053001 (2004).

[44] I. Dorsner and P. Fileviez Pérez, Unification without supersymmetry: Neutrino mass, proton decay and light leptoquarks, Nucl. Phys. B723, 53 (2005).

[45] D. Emmanuel-Costa and R. González Felipe, Minimal string-scale unification of gauge couplings, Phys. Lett. B 623, 111 (2005).

[46] R. Shrock, Variants of the standard model with electroweak-singlet quarks, Phys. Rev. D 78, 076009 (2008).

[47] I. Gogoladze, B. He, and Q. Shafi, New fermions at the LHC and mass of the Higgs boson, Phys. Lett. B 690, 495 (2010).

[48] R. Dermíšek, Insensitive unification of gauge couplings, Phys. Lett. B 713, 469 (2012).

[49] R. Dermíšek, Unification of gauge couplings in the Standard Model with extra vectorlike families, Phys. Rev. D 87, 055008 (2013).

[50] I. Doršner, S. Fajfer, and I. Mustać, Light vectorlike fermions in a minimal su(5) setup, Phys. Rev. D 89, 115004 (2014).

[51] M.-L. Xiao and J.-H. Yu, Stabilizing electroweak vacuum in a vectorlike fermion model, Phys. Rev. D 90, 014007 (2014).

[52] B. Bhattacherjee, P. Byakti, A. Kushwaha, and S. K. Vempati, Unification with vector-like fermions and signals at LHC, J. High Energy Phys. 05 (2018) 090.

[53] J. Schwichtenberg, Gauge coupling unification without supersymmetry, Eur. Phys. J. C 79, 351 (2019).

[54] N. Arkani-Hamed and S. Dimopoulos, Supersymmetric unification without low energy supersymmetry and signatures for fine-tuning at the LHC, J. High Energy Phys. 06 (2005) 073.

[55] V. Barger, J. Jiang, P. Langacker, and T. Li, String scale gauge coupling unification with vector-like exotics and noncanonical u(1)y normalization, Int. J. Mod. Phys. A 22, 6203 (2007)

[56] V. Barger, N. G. Deshpande, J. Jiang, P. Langacker, and T. $\mathrm{Li}$, Implications of canonical gauge coupling unification in high-scale supersymmetry breaking, Nucl. Phys. B793, 307 (2008).

[57] L. Calibbi, L. Ferretti, A. Romanino, and R. Ziegler, Gauge coupling unification, the gut scale, and magic fields, Phys. Lett. B 672, 152 (2009).

[58] I. Donkin and A. Hebecker, Precision gauge unification from extra Yukawa couplings, J. High Energy Phys. 2010, 0944.

[59] C. Liu and Z.-H. Zhao, $\mathrm{Su}(3)$ and the higgs mass from high scale supersymmetry, Commun. Theor. Phys. 59, 467 (2013).

[60] T. Goto and T. Nihei, New constraint on the minimal SUSY GUT model from proton decay, in International Symposium on Supersymmetry, Supergravity and Superstring (2000), pp. 216-228, https://arxiv.org/abs/hep-ph/ 9909251.
[61] J. Hisano, H. Murayama, and T. Yanagida, Probing GUT Scale Mass Spectrum Through Precision Measurements on the Weak Scale Parameters, Phys. Rev. Lett. 69, 1014 (1992).

[62] J. Hisano, T. Moroi, K. Tobe, and T. Yanagida, Limit on the color triplet Higgs mass in the minimum supersymmetric SU (5) model, Mod. Phys. Lett. A 10, 2267 (1995).

[63] J. L. Cardy, Is there a c-theorem in four dimensions?, Phys. Lett. B 215, 749 (1988).

[64] H. Osborn, Derivation of a four dimensional c-theorem for renormaliseable quantum field theories, Phys. Lett. B 222, 97 (1989).

[65] I. Jack and H. Osborn, Analogs of the c-theorem for fourdimensional renormalisable field theories, Nucl. Phys. B343, 647 (1990).

[66] H. Osborn, Weyl consistency conditions and a local renormalisation group equation for general renormalisable field theories, Nucl. Phys. B363, 486 (1991).

[67] O. Antipin, M. Gillioz, J. Krog, E. Mølgaard, and F. Sannino, Standard model vacuum stability and weyl consistency conditions, J. High Energy Phys. 08 (2013) 034.

[68] M. Luo, H. Wang, and Y. Xiao, Two-loop renormalization group equations in general gauge field theories, Phys. Rev. D 67, 065019 (2003).

[69] M. Tanabashi et al., Review of particle physics, Phys. Rev. D 98, 030001 (2018).

[70] I. V. Krive and A. D. Linde, On the vacuum stability problem in gauge theories, Nucl. Phys. B117, 265 (1976).

[71] K. Holland and J. Kuti, How light can the Higgs be?, Nucl. Phys. B, Proc. Suppl. 129, 765 (2004).

[72] K. Holland, Triviality and the Higgs mass lower bound, Nucl. Phys. B, Proc. Suppl. 140, 155 (2005).

[73] P. Gerhold and K. Jansen, The Phase structure of a chirally invariant lattice Higgs-Yukawa model for small and for large values of the Yukawa coupling constant, J. High Energy Phys. 09 (2007) 041.

[74] P. Gerhold and K. Jansen, The Phase structure of a chirally invariant lattice Higgs-Yukawa model-numerical simulations, J. High Energy Phys. 10 (2007) 001.

[75] P. Gerhold and K. Jansen, Lower Higgs boson mass bounds from a chirally invariant lattice Higgs-Yukawa model with overlap fermions, J. High Energy Phys. 07 (2009) 025.

[76] P. Gerhold and K. Jansen, Upper Higgs boson mass bounds from a chirally invariant lattice Higgs-Yukawa model, J. High Energy Phys. 04 (2010) 094.

[77] J. Bulava, P. Gerhold, K. Jansen, J. Kallarackal, B. Knippschild, C. J. D. Lin, K.-I. Nagai, A. Nagy, and K. Ogawa, Higgs-Yukawa model in chirally-invariant lattice field theory, Adv. High Energy Phys. 2013, 875612 (2013).

[78] H. Gies, C. Gneiting, and R. Sondenheimer, Higgs mass bounds from renormalization flow for a simple Yukawa model, Phys. Rev. D 89, 045012 (2014).

[79] H. Gies and R. Sondenheimer, Higgs mass bounds from renormalization flow for a Higgs-top-bottom model, Eur. Phys. J. C 75, 68 (2015). 
[80] A. Eichhorn and M. M. Scherer, Planck scale, Higgs mass, and scalar dark matter, Phys. Rev. D 90, 025023 (2014).

[81] A. Jakovac, I. Kaposvari, and A. Patkos, Scalar mass stability bound in a simple Yukawa-theory from renormalization group equations, Mod. Phys. Lett. A 32, 1750011 (2017).

[82] H. Gies, R. Sondenheimer, and M. Warschinke, Impact of generalized Yukawa interactions on the lower Higgs mass bound, Eur. Phys. J. C 77, 743 (2017).

[83] R. Sondenheimer, Nonpolynomial Higgs interactions and vacuum stability, Eur. Phys. J. C 79, 1 (2019)..

[84] H. Gies and R. Sondenheimer, Renormalization group flow of the Higgs potential, Phil. Trans. R. Soc. A 376, 20170120 (2018).

[85] M. Reichert, A. Eichhorn, H. Gies, J. M. Pawlowski, T. Plehn, and M. M. Scherer, Probing baryogenesis through the Higgs self-coupling, Phys. Rev. D 97, 075008 (2018).

[86] G. G. Ross, Grand Unified Theories (Benjamin/ Cummings, Menlo Park, CA, 1985).

[87] R. Slansky, Group theory for unified model building, Phys. Rep. 79, 1 (1981).

[88] E. Witten, Mass hierarchies in supersymmetric theories, Phys. Lett. 105B, 267 (1981).

[89] D. V. Nanopoulos and K. Tamvakis, SUSY GUTS: 4GUTS: 3, Phys. Lett. 113B, 151 (1982).

[90] S. Dimopoulos and H. Georgi, Solution of the gauge hierarchy problem, Phys. Lett. 117B, 287 (1982).

[91] L. E. Ibanez and G. G. Ross, SU(2)-L $\times$ U(1) Symmetry breaking as a radiative effect of supersymmetry breaking in guts, Phys. Lett. 110B, 215 (1982).

[92] D. G. C. McKeon and C. Zhao, Multiple couplings and renormalization scheme ambiguities, Nucl. Phys. B932, 425 (2018).

[93] T. Banks and A. Zaks, On the phase structure of vector-like gauge theories with massless fermions, Nucl. Phys. B196, 189 (1982).

[94] S. Weinberg, Effective gauge theories, Phys. Lett. B 91, 51 (1980).

[95] L. J. Hall, Grand unification of effective gauge theories, Nucl. Phys. B178, 75 (1981).

[96] H. Gies and L. Zambelli, Asymptotically free scaling solutions in non-Abelian Higgs models, Phys. Rev. D 92, 025016 (2015).
[97] H. Gies and L. Zambelli, Non-Abelian Higgs models: Paving the way for asymptotic freedom, Phys. Rev. D 96, 025003 (2017).

[98] H. Gies, R. Sondenheimer, A. Ugolotti, and L. Zambelli, Asymptotic freedom in $\mathbb{Z}_{2}$-Yukawa-QCD models, Eur. Phys. J. C 79, 101 (2019).

[99] H. Gies, R. Sondenheimer, A. Ugolotti, and L. Zambelli, Scheme dependence of asymptotically free solutions, Eur. Phys. J. C 79, 463 (2019).

[100] N.-P. Chang and J. Perez-Mercader, Eigenvalue conditions and asymptotic freedom of $\mathrm{SO}(N)$ gauge theories, Phys. Rev. D 18, 4721 (1978); , Erratum, Phys. Rev. D 19, 2515 (1979).

[101] D. J. E. Callaway, Triviality pursuit: Can elementary scalar particles exist?, Phys. Rep. 167, 241 (1988).

[102] E. Gildener, Gauge symmetry hierarchies, Phys. Rev. D 14, 1667 (1976).

[103] S. Weinberg, Gauge hierarchies, Phys. Lett. B 82, 387 (1979).

[104] E. Gildener, Gauge symmetry hierarchies revisited, Phys. Lett. 92B, 111 (1980).

[105] M. Luo and Y. Xiao, Two Loop Renormalization Group Equations in the Standard Model, Phys. Rev. Lett. 90, 011601 (2003).

[106] M. E. Machacek and M. T. Vaughn, Two loop renormalization group equations in a general quantum field theory. 1. Wave function renormalization, Nucl. Phys. B222, 83 (1983).

[107] M. E. Machacek and M. T. Vaughn, Two loop renormalization group equations in a general quantum field theory. 2. Yukawa couplings, Nucl. Phys. B236, 221 (1984).

[108] M. E. Machacek and M. T. Vaughn, Two loop renormalization group equations in a general quantum field theory. 3. Scalar quartic couplings, Nucl. Phys. B249, 70 (1985).

[109] M. Luo, H. Wang, and Y. Xiao, Two loop renormalization group equations in general gauge field theories, Phys. Rev. D 67, 065019 (2003).

[110] M. Einhorn and D. Jones, Asymptotic freedom in certain $s o(n)$ and $s u(n)$ models, Phys. Rev. D 96, 055035 (2017). 